\title{
ADAPTIVE FREQUENCY ESTIMATION IN SMART GRID APPLICATIONS: EXPLOITING NONCIRCULARITY AND WIDELY LINEAR ADAPTIVE ESTIMATORS
}

\author{
Yili Xia ${ }^{1}$, Scott C. Douglas ${ }^{2}$, and Danilo P. Mandic ${ }^{1}$ \\ ${ }^{1}$ Imperial College London, U.K. ${ }^{2}$ Southern Methodist University, Dallas, USA \\ \{yili.xia06,d.mandic\}@imperial.ac.uk,douglas@engr.smu.edu
}

Signal processing is a crucial technology for the efficient use of limited and intermittent power resources in the smart grid of the future, and a number of challenges remain to be met. One major issue, as we move towards distributed energy production and use (microgrid) is real time estimation of power quality parameters (frequency, voltages, power factor). The accurate knowledge of frequency is a key parameter of a power system, and its optimal estimation becomes critical in the future smart grid, where the generation, loading and topology are all dynamically updated. In this work, we first consolidate the existing approaches to real-time frequency estimation in a three phase system, and then provide a unified framework for the estimation of the instantaneous frequency in both balanced and unbalanced conditions of a three phase power system. This is achieved by using recent developments in the statistics of complex variables (augmented statistics), by employing the associated widely linear models, and by rigorously accounting for the different degrees of noncircularity associated with various natures of frequency variations in real-world conditions. The usefulness of the proposed framework for frequency tracking in smart grids is illustrated in the context of two major issues in power quality control, namely the tracking of false frequency perturbations in the presence of unbalanced voltage sags (here both synthetic and real-world) and in adaptive frequency tracking in microgrids and islands where there is mismatch between production and consumption.

\section{THE NEED FOR FREQUENCY ESTIMATION IN SMART GRID}

Governments, utilities and consumers are all interested in making the ways we produce and use energy more efficient and sustainable. For the electrical power grid this involves fundamental paradigm shifts as we build a smart grid, adopt more renewable energy sources, and promote more energy efficient practices. A smart grid delivers electricity from suppliers to users using digital technology and has a number of properties, including incorporating all forms of energy generation and storage, using sensor information, enabling active participation by end users, being secure and reliable, and using optimization and control to make decisions [1]. This will require the interplay between sensor networks, generation systems, and the power grid, with key technologies from signal processing.

It is estimated that the financial loss due to outages in the US economy approaches USD $\$ 45.7$ billion annually, with power quality issues costing USD $\$ 6.7$ billion annually $[2,3]$. Among them, voltage sags, that is, an increase in load current over up to few hundred cycles, are the most frequent problem [4] that severely affects medical centres, semiconductor plants, and broadcasting stations, among others [5]. Voltage sags are typically followed by frequency variations and occur due to switching between the main grid and microgrids, short circuits, motor starting, transformer inrush, fast reclosing of circuit breakers, unexpectedly large or fast change in the load, malfunction of electronic protection components, or when the scale of the problem causes a large number of alarms which saturate the alarm processing capability of the control center [6].

In a power system, unexpected frequency variations from the nominal value can trigger abnormal system conditions and disturbances, and fast and accurate frequency estimation has recently attracted much attention $[7,8,9,10,11]$. Deriving the system frequency from a single phase is a non-unique problem [12], and for robust frequency estimation it is desired to simultaneously consider all the three phase voltages. A classic approach is to use Clarke's $\alpha \beta$ transformation to obtain a complex-valued signal from the three phase voltages [13], and derive system frequency from the phase of the transformed signal.

This work addresses adaptive tracking of system frequency in a 3-phase system, and proposes next generation solutions for the identification and troubleshooting in the following events that lead to frequency deviations.

- Imbalance in the generation (G) and load (L). In smart grid, the system will frequently switch between the main grid (MG) and microgrids $(\mu \mathrm{G})$, with parts of the system completely switching off the MG for the prolonged periods of time (islanding). The system frequency rises for $\mathrm{G}>\mathrm{L}$ and decreases for $\mathrm{L}<\mathrm{G}$.

- Single and dual phase faults. The system frequency is derived from the relationship between the three phase 
voltages (using Clarke's transform). Faults in one or two phases and voltage sags (sudden drop in voltage for a short period of time) will cause an incorrect frequency estimate and an alarm spread through the system, although the actual system frequency is correct.

- Dual character of load-supply. The key idea behind smart grid is to have dynamic loads and dual loadgenerator devices, such as for instance Plug-in Electric Vehicles (PEV)s, which can give the energy back to the grid in the case of emergency. This will cause problems with reactive power, whose drifting is responsible for oscillations of power levels and harmonics in frequency.

- Harmonics. Some loads (power supplies, motors, pumps, heating elements) that have nonlinear V-I characteristics introduce harmonics, which are slowly floating and may not be integer multiplies of system frequency. They cause resonance in the system and lead to overheating of transformers and significant increase in currents [14]. In addition, switching on the shunt capacitors for reactive energy compensation causes strong transients and harmonics that are damaging to some equipment.

- Transient stability issues. Faults and short circuits make the system unstable, and actions such as shedding loads (or generators) that are needed to mitigate the problem must occur within a short period of time, typically $100 \mathrm{~ms}$ [15]. Accurate frequency estimation is required to make this possible.

Although some of the events leading to frequency changes in the system could be dealt with if detected in time, current systems do not have sufficient information about the state at the distribution end to do so. This applies particularly to problems related to the management of single and dual phase faults, short duration voltage sags, and reactive power, causing damaging events, such as harmonics, false alarms, and slow response to critical events.

The Clarke's transform based complex domain solutions for frequency tracking include phase locked loops (PLL) [16], least squares methods [17], Kalman filtering [18], and demodulation methods [19]. Recently, adaptive tracking algorithms based on the minimisation of mean square error have become a standard, as they are naturally suited to deal with noise, harmonics, and nonstationary environments [7, 17, 20]. However, as discussed in [21], unbalanced events make it difficult to calculate phase angle - the complex-valued signal obtained from an unbalanced three-phase voltage source is represented as an orthogonal sum of positive and negative sequences (SCT - see (2)). Standard complex linear adaptive filters can only cater for the positive sequences, whereas the negative sequences introduce a modelling error that oscillates at twice the system frequency [22, 23, 24].
To this end, we introduce a robust framework for adaptive and unbiased frequency estimation under unbalanced system conditions, a typical case in microgrids, coupled microgrids and power islands.

- We first analyse the complex-valued signal, obtained by the $\alpha \beta$ transformation of a three-phase power system, and illustrate the suitability of complex valued filters in this context by illuminating their magnitude-phase relationship and tracking abilities.

- We then address the geometry of learning to leverage between magnitude-only and phase-only adaptive tracking, and emphasize the trade off between bias and variance of such adaptive frequency estimators.

- It is further illustrated that under unbalanced system conditions the $\alpha \beta$-transformed complex voltage signal is second order noncircular (improper), for which current, strictly linear, complex valued adaptive estimators are suboptimal.

- A second-order optimal adaptive widely linear frequency estimator is next addressed, and is shown to cater for both the balanced and unbalanced system conditions and to produce unbiased estimates with greatly reduced variance as compared with standard approaches, asymptotically approaching the CramerRao Lower Bound (CRLB) for high signal to noise ratios.

\section{FROM THE HIERARCHICAL GRID TO SMART GRID, MI- CROGRIDS, AND ENERGY ISLANDS}

Electric distribution networks are undergoing wholesale changes both from the generation and the user (load) sides. Generation, historically aggregated into large power plants and far from the user, is beginning to be moved towards being located at the distribution level, and based on renewable sources, that is, intrinsically intermittent [25]. This will require enhanced flexibility in order to accommodate islanding and micro-grids [26, 27]. Major challenges are envisaged to be the management of largely increased load levels and the synergy between loads and supplies. For instance, charging large number of Plug-In Electric Vehicles (PEVs) in a residential area is likely to lead to unbalanced system conditions - causing increased levels of harmonics and frequency deviations that are potentially damaging to households electronic and electrical equipment. On the other hand, PEVs are designed to discharge their batteries quickly (e.g. when accelerating) and may be used when in the vehicle-to-grid (V2G) mode to feed the energy back into the grid, in order to mitigate power shortage and system imbalances.

The idea behind smart distributed grids and microgrids is to balance as much as possible locally between production 


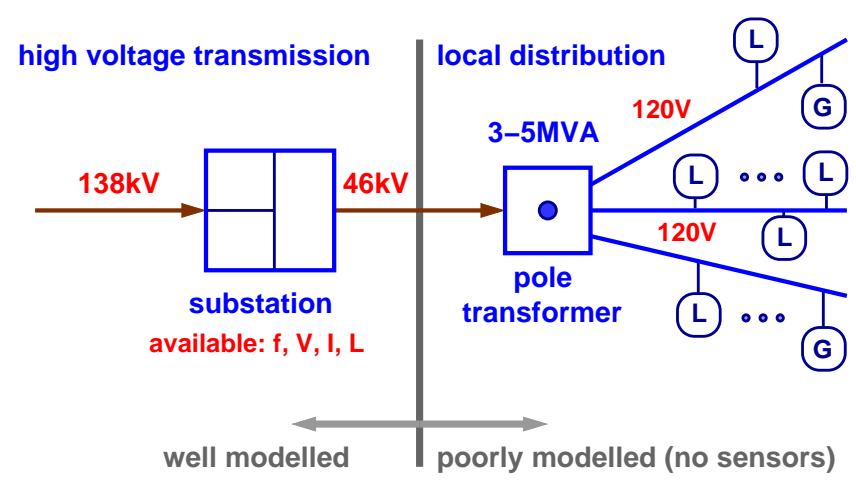

Fig. 1. Block diagram of power grid. The transmission side is well understood and the measurements of voltage, frequency, current and load are available. The distribution side does not have enough sensors and the transmission side does not have sufficient information about local behaviour. Various loads (L) and small generators $(\mathrm{G})$ contribute to the variability of power quality.

and consumption. However, the nature of intermittent renewable sources will inevitably lead to frequent system imbalance. For instance, the variability of wind and solar power seldom correlates with the load variability. There is evidence of difficulty in maintaining system balance due to wind variability [28]. Accurate and fast frequency tracking is a prerequisite to the system responding quickly to such problems, and signal processing is certain to play a large role in dealing with the complexity and uncertainty associated with frequency estimation in smart grid. In this context, stochastic models are a natural choice.

Fig. 1 shows a simplified diagram of the transmission and distribution part of the grid, illustrating the available loads $(\mathrm{L})$, generators $(\mathrm{G})$, and measurements $(\mathrm{M})$ at both the substation and consumer level. One of the main problems in frequency estimation is that at present the transmission side of the grid does not have sufficient information about the system behaviour at the distribution level, in order to incorporate it into the operator model.

The high voltage transmission line (e.g. $138 \mathrm{kV}$ ) is transformed down to $46 \mathrm{kV}$ at the substation, with each substation typically having 3-5 branches (called circuits). At present, Phasor Measurement Units (PMU), which use synchronised measurements based on the Global Positioning System (GPS), are mostly deployed at the substations, and forward synchronised data to the main station. The voltage signals are obtained at 48 samples/cycle, that is, approximately at the sampling frequency of $1000 \mathrm{~Hz}$. The measurements available at the substation are the three phase voltages, currents, system frequency, and loads. This provides the operator model with the information about the state of the grid, and this part is well modelled.

The progress towards microgrids, coupled microgrids,

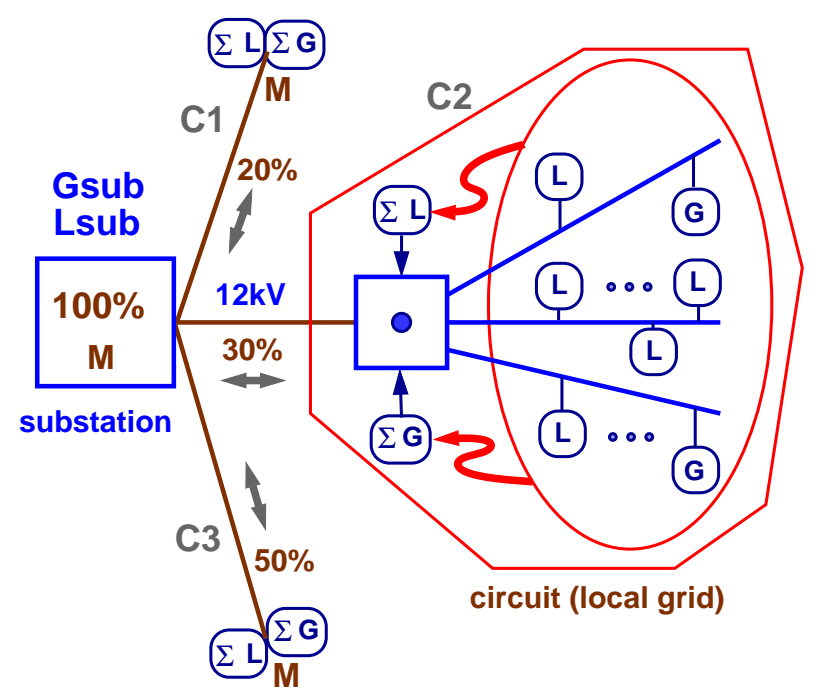

Fig. 2. Nodal Estimation. The load (L) and generators (G) operate locally. Every substation has 3-5 circuits (main branches). The sum of all the circuit loads $\Sigma \mathrm{L}$ and all the generation $\Sigma G$ equals that of the substation. Symbol $M$ indicates where measurements area available. All circuits shown are three phase. In case of G-L mismatch in one of the circuits, this can be compensated from the generation in another circuit or from the main grid.

and islanding will introduce numerous problems in power quality, including frequency estimation, and will require more metering devices at the distribution and consumer level.

The roles of the main station and substation are [6]:

- The main station collects and manages PMU data and real-time switching information from the substations, diagnoses wide area faults and issues control orders (switching off and blocking) to the substations;

- The substations collect and forward the local data to the main station and execute control sequences received from the main station.

The so called Nodal Estimation, depicted in Fig. 2, illustrates these issues - a substation with three circuits (C1-C3) taking respectively $20 \%, 30 \%$ and $50 \%$ of the generation, and a zoom in into the local grid (from the pole transformer down to customer). In classical distribution systems, voltage is normally controlled only at the entry point, however, modern systems are not designed vertically and have both the consumption and production at the distribution level. Wind energy is more likely to be connected at the generation level, whereas photovoltaic sources that produce $1 \sim 2 \mathrm{~kW}$ are often located at the customer's site. They offset the connection load, and can also feed the energy back into the grid [29]. The operation of the power system at a constant frequency is maintained 


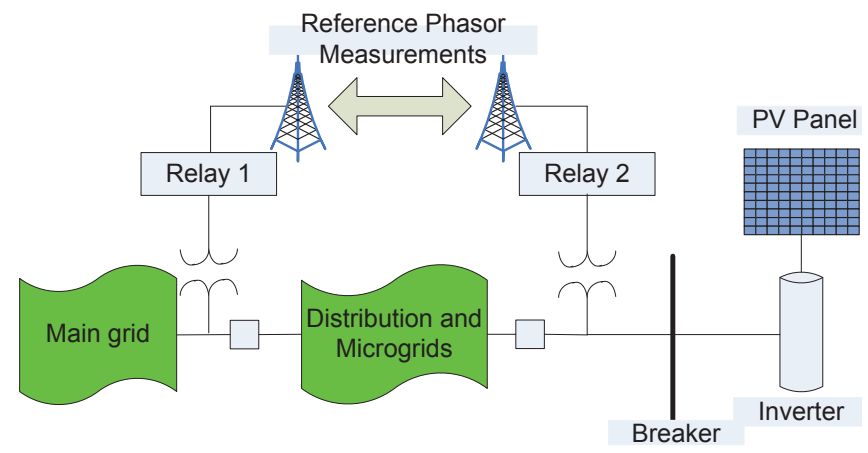

Fig. 3. Islanding regulation, using relays and inverters.

by regulating the balance between generation and load in real time.

\section{MICROGRIDS AND ISLANDS}

Microgrids are small connected clusters within the main grid, and can operate in parallel to the grid or isolated (as an island). They respond to fault events autonomously and based on only local information, switching to the islanding mode for faults (dynamic islanding), such as large voltage sags and power outages. They facilitate the implementation of key Smart Grid functions, such as load control, reliability and self-healing, but this comes at a cost of constant switching, as they will disconnect when the power quality of the main grid is below certain standards. It is desired that the microgrid seamlessly changes its mode of operation between an island (energy source for local loads) and a grid resource.

This also facilitates a greater use of renewables, but requires local stability and constant monitoring of large circulating reactive currents between sources, together with voltage vs. power droop control. Fig. 3 (adopted from [15]) illustrates the principle of connecting a Photo-Voltaic (PV) generator to the grid through the circuit breakers and relays. The microgrid operation is summarised in the following.

- In normal operation, the loads in microgrid receive power from both the main grid and local generators;

- When the grid power is lost, the microgrid dynamically transforms into the islanding mode;

- If the microgrid was taking the energy from the grid, local generation increases the available power - resulting in a temporary drop in frequency;

- If the islanding event occurred when the microgrid was exporting power to the main grid, the system frequency will increase;

- If the power mismatch between the source and load is small, this is difficult to detect; so for instance, an invertor that constantly attempts to shift its local frequency would indicate a power island.

\section{VOLTAGE SAGS}

Voltage sags refer to a temporary drop in the one or two phase voltages for several hundreds milliseconds [27]. Despite their short duration they are harmful to a range of equipment, including computers, adjustable speed devices and three-phase loads. A voltage sag is defined by the IEEE Standard 11591995 as a "decrease in root mean square (RMS) voltage at the power frequency for durations from 0.5 cycles to 1 minute". It is measured by the percentage of the nominal voltage, so that a sag of $60 \%$ is equivalent to $60 \%$ of nominal root-meansquare (RMS) voltage. A voltage dip is the amount by which the voltage drops from its nominal value, that is, a voltage dip of $40 \%$ is equivalent to a voltage sag of $60 \%$. The voltage sags are detected when the RMS magnitude drop exceeds a predefined threshold, typically $90 \%$ of the nominal voltage.

Single phase faults and sags typically occur in rural distribution systems. In three-phase systems, the other two phase voltages go into a swell (increase in voltage) in order to maintain the required power by the load, even in the case of a single phase voltage sag. Three-phase sags can be symmetric, for instance, when starting a large motor in an industrial plant, or unbalanced as when energising a large transformer. In all cases there is a large and sudden drop in phase voltage(s), followed by a slow recovery. The kind of sag experienced by the load also depends on whether it is connected in the star or delta configuration.

Voltage sags are multifaceted phenomena, yet since there are typically one or two transformers between the phase fault and the meter (or load) they are rarely recorded at the level at they occurred. Voltage sags that occur at the higher voltage side are spread to the lower voltage systems through transformers, and sags and faults that appear at one circuit of the low voltage side will also affect the other circuits at the same substation (see Fig. 2). System frequency is estimated from the three phase voltages, and therefore, accurate and fast estimation of voltage sags is a prerequisite.

\section{DEALING WITH THE THREE-PHASE VOLTAGES - CLARKE'S TRANSFORM}

The voltages of a three phase power system in a noise-free environment can be represented in the discrete time form as

$$
\begin{aligned}
& v_{a}(k)=V_{a}(k) \cos (\omega k \triangle \mathrm{T}+\phi) \\
& v_{b}(k)=V_{b}(k) \cos \left(\omega k \triangle \mathrm{T}+\phi-\frac{2 \pi}{3}\right) \\
& v_{c}(k)=V_{c}(k) \cos \left(\omega k \triangle \mathrm{T}+\phi+\frac{2 \pi}{3}\right)
\end{aligned}
$$

where $V_{a}(k), V_{b}(k), V_{c}(k)$ are the peak values of each phase voltage component at time instant $k, \triangle \mathrm{T}$ the sampling interval, $\phi$ the phase of fundamental component, and $\omega=2 \pi f$ the angular frequency of the voltage signal, with $f$ being the system frequency. 
A direct analysis of the individual phase voltages in (1) is not practical for the estimation of power quality parameters - single phase techniques are limited and it is difficult to select the most representative phase, since 6 different phase voltages exist in a three phase system when also line-to-line voltages are considered. We therefore need a system solution operating simultaneously on all the three phases, and attempts have been made to introduce invertible transformations which would give physical meaning to the estimation in a transform domain.

The symmetrical component transformation (SCT) [30] can determine the type of voltage sag, and is given by

$$
\left[\begin{array}{l}
\bar{V}_{0} \\
\bar{V}_{1} \\
\bar{V}_{2}
\end{array}\right]=\frac{1}{3}\left[\begin{array}{ccc}
1 & 1 & 1 \\
1 & a & a^{2} \\
1 & a^{2} & a
\end{array}\right]\left[\begin{array}{l}
\bar{V}_{a} \\
\bar{V}_{b} \\
\bar{V}_{c}
\end{array}\right]
$$

where e.g. $\bar{V}_{a}=V_{a} e^{\jmath \Phi_{a}}$ is the complex magnitude for phase $\mathrm{a}, v_{a}(k)=\sqrt{2} V_{a} \cos \left(\omega_{0} k+\Phi_{a}\right)$ is the instantaneous voltage, $a=e^{\jmath \frac{2 \pi}{3}}$ corresponds to a phase angle shift, and $\bar{V}_{i}=V_{i} e^{\jmath \Phi_{i}}$. The transformed quantities are: $\bar{V}_{0}$ - the zero sequence, $\bar{V}_{1}$ positive sequence, and $\bar{V}_{2}$ - negative sequence. In physical terms, the positive sequence voltage reflects the energy transfer between generators and consumers; the negative-sequence and zero-sequence components indicate imbalance between the three voltages; the negative sequence propagates from the fault to the equipment terminals; the zero-sequence is in many cases blocked by the transformers. A voltage dip is characterised by a relationship between the positive sequence voltage $\bar{V}_{1}$ and the negative sequence voltage $\bar{V}_{2}$.

The SCT is based on a phasor representation (no notion of time), and is thus not suitable for the analysis of transients - these would need a time domain representation whose high resolution is guaranteed by a high sampling frequency of the phase voltages (e.g. $1000 \mathrm{~Hz}$ as opposed to the $50 \mathrm{~Hz}$ system frequency). To this end, Clarke's transform relates phase-toneutral voltages and phase voltages using the following mapping

$$
\left[\begin{array}{l}
V_{a} \\
V_{b} \\
V_{c}
\end{array}\right]=\left[\begin{array}{ccc}
1 & 0 & 1 \\
-\frac{1}{2} & \frac{\sqrt{3}}{2} & 1 \\
-\frac{1}{2} & -\frac{\sqrt{3}}{2} & 1
\end{array}\right]\left[\begin{array}{l}
V_{\alpha} \\
V_{\beta} \\
V_{0}
\end{array}\right]
$$

or conversely, the time-dependent three-phase voltage is transformed by the orthogonal $\alpha \beta 0$ transformation matrix [13] into a zero-sequence $v_{0}$ and the direct and quadratureaxis components, $v_{\alpha}$ and $v_{\beta}$, as

$$
\left[\begin{array}{l}
v_{0}(k) \\
v_{\alpha}(k) \\
v_{\beta}(k)
\end{array}\right]=\sqrt{\frac{2}{3}}\left[\begin{array}{ccc}
\frac{\sqrt{2}}{2} & \frac{\sqrt{2}}{2} & \frac{\sqrt{2}}{2} \\
1 & -\frac{1}{2} & -\frac{1}{2} \\
0 & \frac{\sqrt{3}}{2} & -\frac{\sqrt{3}}{2}
\end{array}\right]\left[\begin{array}{c}
v_{a}(k) \\
v_{b}(k) \\
v_{c}(k)
\end{array}\right]
$$

The factor $\sqrt{2 / 3}$ is used to ensure that the system power is invariant under this transformation. When $V_{a}(k), V_{b}(k), V_{c}(k)$ are identical, $v_{0}(k)=0, v_{\alpha}(k)=A \cos (\omega k \Delta \mathrm{T}+\phi)$ and $v_{\beta}(k)=A \cos \left(\omega k \Delta \mathrm{T}+\phi+\frac{\pi}{2}\right)$, with a constant amplitude
$A$, while $v_{\alpha}(k)$ and $v_{\beta}(k)$ are the orthogonal coordinates of a point whose position is time variant at a rate proportional to the system frequency. In practice, only the $v_{\alpha}$ and $v_{\beta}$ parts are used in the modelling, known as the $\alpha \beta$ transformation [19].

The resulting complex voltage signal $v(k)$ is given by

$$
v(k)=v_{\alpha}(k)+\jmath v_{\beta}(k)
$$

and will be the basis for the analysis in this work. There is no loss in information in using this representation, and this complex time-domain voltage also serves as the desired signal in adaptive frequency estimation and can be calculated iteratively from (in a balanced system condition)

$$
v(k+1)=A e^{\jmath(\omega(k+1) \Delta \mathrm{T}+\phi)}=v(k) e^{\jmath \omega \Delta \mathrm{T}}
$$

\section{THE ADAPTIVE FREQUENCY ESTIMATION FRAME- WORK: COMPLEX CIRCULARITY, STRICTLY LINEAR AND WIDELY LINEAR MODELS}

We shall now introduce a general framework for both the strictly linear and widely linear complex-valued frequency estimation, based on the complex voltage in (6).

The strictly linear framework employs the recently introduced Least Mean Magnitude Phase (LMMP) algorithm [31] to leverage between the standard, error power based, Complex Least Mean Square (CLMS) [32] and phase-only estimation realised by the Least Mean Phase (LMP) algorithm [33]. It is shown that all the strictly linear algorithms exhibit bias and large oscillations of the mean squared error, with the LMP best suited for frequency estimation in terms of the bias and LMMP in terms of the steady state properties. This is not unexpected - LMP was designed specifically for phase minimisation and the frequency estimate is derived from the phase of the complex $\alpha \beta$ voltage in (6), whereas LMMP is general enough to cater for the evolution of error variance.

Next, for adaptive estimation of complex signals with second order noncircular (improper, rotation-dependent) distributions, it is shown that the covariance matrix $\mathrm{C}_{\mathbf{x x}}=E\left[\mathbf{x x}^{H}\right]$, does not contain sufficient information, and the pseudocovariance matrix $\mathrm{P}_{\mathbf{x x}}=E\left[\mathbf{x} \mathbf{x}^{T}\right]$ should also be taken into account to describe the complete second order behaviour of the complex random vector $\mathbf{x}$ [34], [35]. In practice, this is achieved by virtue of the widely linear modelling $[34,36]$, where both $\mathbf{x}$ and its complex conjugate $\mathbf{x}^{*}$ are combined into the augmented input $\mathbf{x}_{a}=\left[\mathbf{x}^{T}, \mathbf{x}^{H}\right]^{T}$. The widely linear Augmented Complex Least Mean Square (ACLMS) algorithm [37, 36] is then exploited in adaptive frequency estimation of the generality of system conditions - both balanced (circular) and unbalanced (noncircular). The strictly linear models (CLMS, LMP, LMMP) are shown to provide biased and non-minimum variance solutions, while the widely linear ACLMS produced an unbiased and minimum variance solution, asymptotically approaching the theoretical performance bound - the Cramer Rao Lower Bound (CRLB). 

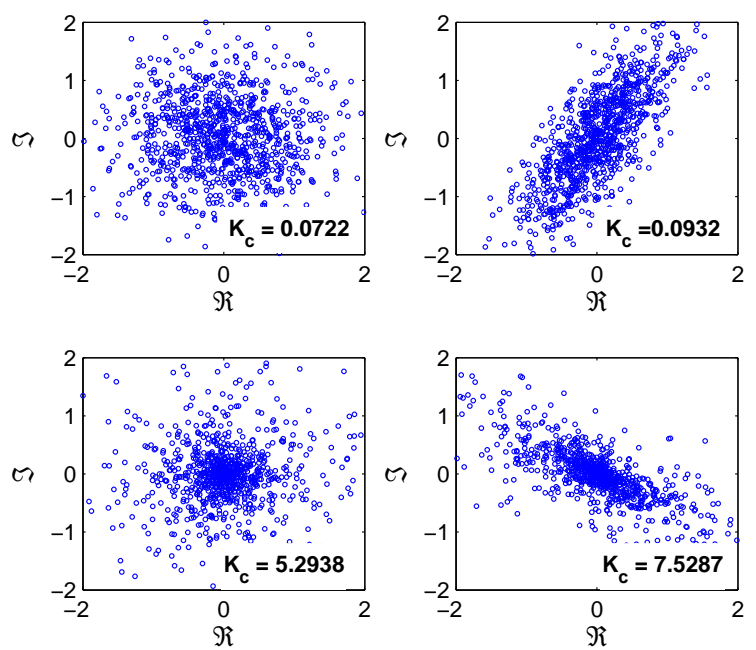

Fig. 4. Scatter plots of complex white noise realisations. Top row: circular Gaussian noise (left) and noncircular Gaussian noise $(\eta=0.81)$ (right). Bottom row: circular Laplacian noise (left) and noncircular Laplacian noise $(\eta=0.81)$ (right). The circularity measure $\eta$ is defined in (15). The kurtosis values $K_{c}$ are given for each case.

\section{COMPLEX CIRCULARITY AND WIDELY LINEAR MOD- ELLING}

Complex circularity is a property of probability density functions, whereby the distribution of a complex random variable $\mathbf{x}$ and its rotation $e^{\jmath \varphi} \mathbf{x}$ are equal for any rotation angle $\varphi$. Examples of circular and noncircular signals, together with their kurtosis values, are given in Fig. 4, where the symbol $\Re$ denotes the real and $\Im$ the imaginary part of a complex number. Most real world complex-valued signals are noncircular. It is not practical to quantify noncircularity from the probability distributions, however, and we usually consider second order circularity (properness) and second order noncircularity (improperness), which are derived from the powers in the real and imaginary part, based on covariance functions.

Widely linear model. Consider a real-valued conditional mean squared error (MSE) estimator

$$
\hat{y}=E[y \mid \mathbf{x}]
$$

which estimates the signal $y$ in terms of another observation $\mathbf{x}$. For zero mean, jointly normal $y$ and $\mathbf{x}$, the optimal solution is the linear estimator given by

$$
\hat{y}=\mathbf{x}^{T} \mathbf{h}
$$

where $\mathbf{h}=\left[h_{1}, \ldots, h_{L}\right]^{T}$ is a vector of fixed filter coefficients, $\mathbf{x}=\left[x_{1}, \ldots, x_{L}\right]^{T}$ the regressor vector, and $(\cdot)^{T}$ the vector transpose operator.
In the standard, strictly linear estimation in the complex domain, it is assumed that we can use the same form of estimator, leading to the standard complex linear minimum mean square error (MMSE) estimator in the form

$$
\hat{y}=\hat{y}_{r}+\jmath \hat{y}_{i}=\mathbf{x}^{T} \mathbf{h}
$$

where $\jmath=\sqrt{-1}$ and subscripts $r$ and $i$ denote respectively the real and imaginary parts of a complex variable. Since both the real and imaginary parts of complex variables are real, we also have

$$
\hat{y}_{r}=E\left[y_{r} \mid \mathbf{x}_{r}, \mathbf{x}_{i}\right], \quad \hat{y}_{i}=E\left[y_{i} \mid \mathbf{x}_{r}, \mathbf{x}_{i}\right]
$$

and a more general form of (9) becomes

$$
\hat{y}=E\left[y_{r} \mid \mathbf{x}_{r}, \mathbf{x}_{i}\right]+\jmath E\left[y_{i} \mid \mathbf{x}_{r}, \mathbf{x}_{i}\right]
$$

Substitute $\mathbf{x}_{r}=\left(\mathbf{x}+\mathbf{x}^{*}\right) / 2$ and $\mathbf{x}_{i}=\left(\mathbf{x}-\mathbf{x}^{*}\right) / 2$ J to arrive at

$$
\hat{y}=E\left[y_{r} \mid \mathbf{x}, \mathbf{x}^{*}\right]+\jmath E\left[y_{i} \mid \mathbf{x}, \mathbf{x}^{*}\right]=E\left[y \mid \mathbf{x}, \mathbf{x}^{*}\right]
$$

leading to the widely linear estimator for complex valued data

$$
\hat{y}=\mathbf{h}^{T} \mathbf{x}+\mathbf{g}^{T} \mathbf{x}^{*}=\mathbf{x}^{T} \mathbf{h}+\mathbf{x}^{H} \mathbf{g}=\mathbf{x}^{T}(k) \mathbf{w}^{a}(k)
$$

where $\mathbf{h}$ and $\mathbf{g}$ are complex-valued coefficient vectors. In practice, the widely linear estimate in (13) is based on a regressor vector produced by concatenating the input vector $\mathbf{x}$ with its conjugate $\mathbf{x}^{*}$, to give an augmented input vector $\mathbf{x}^{a}=\left[\mathbf{x}^{T}, \mathbf{x}^{H}\right]^{T}$, together with the corresponding augmented coefficient vector $\mathbf{w}^{a}=\left[\mathbf{h}^{T}, \mathbf{g}^{T}\right]^{T}$.

Augmented complex statistics. The $2 L \times 2 L$ augmented covariance matrix, corresponding to the widely linear model in (13), now becomes $[35,38,36]$

$$
\mathbf{C}_{\mathbf{x x}}^{a}=E\left[\begin{array}{c}
\mathbf{x} \\
\mathbf{x}^{*}
\end{array}\right]\left[\mathbf{x}^{H} \mathbf{x}^{T}\right]=\left[\begin{array}{ll}
\mathbf{C}_{\mathbf{x x}} & \mathbf{P}_{\mathbf{x x}} \\
\mathbf{P}_{\mathbf{x x}}^{*} & \mathbf{C}_{\mathbf{x x}}^{*}
\end{array}\right]
$$

and contains the full second order statistical information. From (14), it is clear that the covariance matrix, $\mathbf{C}_{\mathbf{x x}}=$ $E\left[\mathbf{x x}^{H}\right]$, alone does not have sufficient degrees of freedom to describe full second order statistics, and in order to make use of all the available second order information we also need to consider the pseudocovariance matrix, $\mathbf{P}_{\mathbf{x x}}=E\left[\mathbf{x x}^{T}\right]$. Processes with the vanishing pseudocovariance, $\mathbf{P}_{\mathbf{x x}}=\mathbf{0}$, are termed second order circular (or proper). Therefore, the widely linear estimator in (13) is optimal for the generality of complex signals (both proper and improper), and simplifies into the strictly linear model $(\mathbf{g}=\mathbf{0})$ for proper data.

Index of second order noncircularity (improperness). The degree of improperness can be calculated using the circularity index, given by [39]

$$
\eta=\frac{\left|\tau_{x}\right|^{2}}{\sigma_{x}^{2}}
$$

where $\sigma_{x}^{2}=E\left[x(k) x^{*}(k)\right]$ is the variance of the signal $x$, and $\tau_{x}^{2}=E\left[x(k) x^{T}(k)\right]=E\left[\left|x^{2}(k)\right|\right]$ is the absolute value of the 


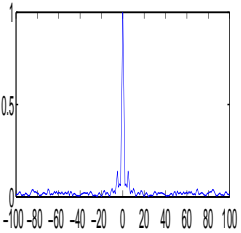

Covarance

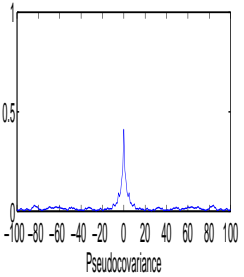

Psecubowaralance

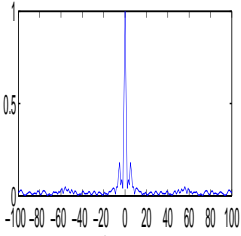

Covalanoe

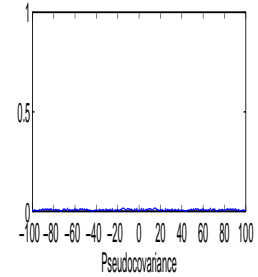

Pseddowowalane

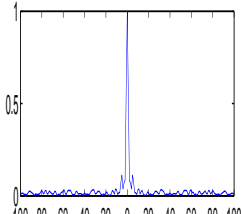

Covarance

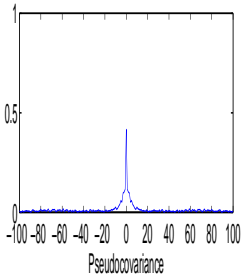

Fig. 5. Widely linear autoregressive modelling of the noncircular Ikeda signal. Top row: covariances, Bottom row: pseudocovariances of (left) the original Ikeda signal, (middle) the strictly linear AR model of Ikeda signal, (right) the widely linear AR model of Ikeda signal.

pseudovariance of $x$. This way, $\eta \in[0,1]$, the value of 0 indicating that $x(k)$ is second order circular (proper), otherwise indicating a second order noncircular (improper) $x(k)$. The angle $\theta=\arg (\eta(x))$ indicates the orientation of the complex distribution - for a purely circular signal, $\eta=0$, and $\theta$ does not provide additional information about the distribution. For graphical interpretation, imagine an ellipse (centred in the complex plane) of eccentricity $\epsilon$ and orientation $\alpha$, such that $\eta=\epsilon^{2}$ and $\theta=2 \alpha$ [39]. For circular signals, $\epsilon=0$, indicating a circle, while for the extreme case of $\epsilon=1$ the ellipse becomes elongated with a maximal major axis and minor axis of length zero.

Widely linear autoregressive modelling. Based on the widely linear model in (13), the widely linear autoregressive model (WLAR) can be written as

$y(k)=\mathbf{h}(k) \mathbf{x}(k)+\mathbf{g}(k) \mathbf{x}^{*}(k)+n(k), \quad n \sim \mathcal{N}\left(0, \tau_{x}^{2}, \sigma_{x}^{2}\right)$

for which the coefficients are obtained from the widely linear Yule-Walker equations, given by [40]

$$
\left[\begin{array}{l}
\mathbf{h}^{*} \\
\mathbf{g}^{*}
\end{array}\right]=\left[\begin{array}{cc}
\mathbf{C} & \mathbf{P} \\
\mathbf{P}^{*} & \mathbf{C}^{*}
\end{array}\right]^{-1}\left[\begin{array}{c}
\mathbf{c} \\
\mathbf{p}^{*}
\end{array}\right]
$$

Fig. 5 illustrates the ability of the WLAR model to explain both the covariance and pseudocovariance of the nonlinear and noncircular (chaotic) Ikeda signal. Notice that the standard AR model was not able to explain the pseudocovariance. The advantage of widely linear estimation over strictly linear estimation can be quantified by the difference between the mean square errors of a strictly linear estimator, $e_{L}^{2}$, and that

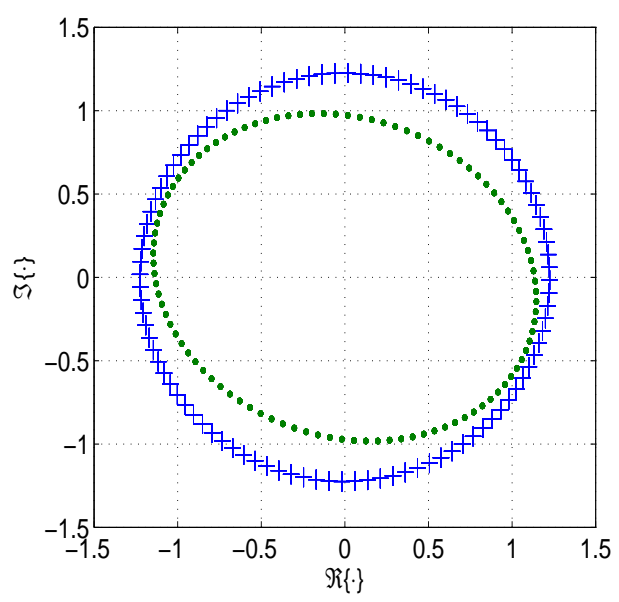

Fig. 6. Circularity via the "real-imaginary" scatter plot in the complex plane. The circle denoted by ' + ' corresponds to a circular complex-valued signal $v(k)$ in a balanced system where $V_{a}(k), V_{b}(k), V_{c}(k)$ are identical at 1-p.u. (per unit) The ellipse denoted by ' ' corresponds to a noncircular $v(k)$ obtained in an unbalanced system condition with $V_{a}(k)=1$ p.u, $V_{b}(k)=0.9$-p.u. and $V_{b}(k)=0.7$-p.u.

of a widely linear estimator, $e_{W L}^{2}$ [34], given by

$$
\begin{aligned}
\delta e^{2} & =e_{L}^{2}-e_{W L}^{2} \\
& =\left[\mathbf{p}-\mathbf{P}_{\mathbf{x x}}^{*} \mathbf{C}_{\mathbf{x x}}^{*-1} \mathbf{c}^{*}\right]^{H}\left[\mathbf{C}_{\mathbf{x x}}-\mathbf{P}_{\mathbf{x x}} \mathbf{C}_{\mathbf{x x}}^{*-1} \mathbf{P}_{\mathbf{x x}}^{*}\right]^{-1} \\
& \cdot\left[\mathbf{p}-\mathbf{P}_{\mathbf{x x}}^{*} \mathbf{C}_{\mathbf{x x}}^{*-1} \mathbf{c}^{*}\right]
\end{aligned}
$$

where $\mathbf{c}=E\left[y^{*} \mathbf{x}\right]$ and $\mathbf{p}=E[y \mathbf{x}]$. Due to the positive definiteness of the matrix $\left[\mathbf{C}_{\mathbf{x x}}-\mathbf{P}_{\mathbf{x x}} \mathbf{C}_{\mathbf{x x}}^{*-1} \mathbf{P}_{\mathbf{x x}}^{*}\right]$, the term $\delta e^{2}$ is always nonnegative. This means that the widely linear estimator has performance advantage over the strictly linear estimator for improper data, whereas their performances on proper data are identical.

Voltage sags and noncircularity. It is now possible to relate the complex circularity with the distribution of the $\alpha \beta$ voltage $v(k)$ from (5) in balanced and unbalanced system conditions. Fig. 6 shows amplitude distribution diagrams for a balanced case and a voltage sag event; when the phase voltages exhibit dips or transients (a sag event), $V_{a}(k), V_{b}(k), V_{c}(k)$ are not identical, and samples of $v(k)$ are located on an ellipse, as illustrated by the ellipse denoted by ' $'$ '. For a balanced system, the distribution of $v(k)$ remains on a circle.

In addition, as shown later in the article, by accounting for the notion of circularity, it is possible in principle to identify the type of the voltage sag and its parameters within a quarter of the frequency cycle.

\section{FREQUENCY ESTIMATION MODELS IN BALANCED AND UNBALANCED THREE PHASE SYSTEMS}

The current state-of-the art is based on the $\alpha \beta$ voltage in (5) and the subsequent application of the complex least 
mean square (CLMS) algorithm. Stochastic gradient algorithms aim to minimise the instantaneous error power, $\mathcal{J}=e(k) e^{*}(k)=|e(k)|^{2}$, using a gradient descent based update of the filter coefficient vector $\mathbf{w}(k)$, in the form

$$
\mathbf{w}(k+1)=\mathbf{w}(k)-\mu e(k) \nabla_{\mathbf{w}} \mathbf{x}(k)
$$

where $\mu$ is a small positive learning rate, $e(k)$ the output error of the filter, and $\mathbf{x}(k)$ the filter input vector (regressor vector). Notice that for the modelling of the $\alpha \beta$ voltage in (5), we only need a single filter coefficient - all the subsequent models will be presented in a single coefficient format.

The strictly linear CLMS model. The CLMS-based model for frequency estimation is given by [17]

$$
\begin{aligned}
\hat{v}(k+1) & =w(k) v(k) \\
e(k) & =v(k+1)-\hat{v}(k+1) \\
w(k+1) & =w(k)+\mu e(k) v^{*}(k)
\end{aligned}
$$

where the filter weight $w(k)$ estimates the phasor $e^{\jmath \omega \Delta \mathrm{T}}$ in (6), $\hat{v}(k+1)$ is the estimate of $v(k+1)$, and the estimated system frequency is derived from

$$
\hat{f}(k)=\frac{1}{2 \pi \Delta \mathrm{T}} \sin ^{-1}(\Im(w(k)))
$$

that is, from the instantaneous value of the filter coefficient.

The strictly linear Least Mean Phase (LMP) model. Observe from (6) that the instantaneous system frequency is obtained based on the phase information in $e^{\jmath \omega \Delta \mathrm{T}}$, and thus the CLMS may be suboptimal, since it is primarily the phase rather than the magnitude that conveys useful information. The complex least-mean-phase (LMP) algorithm [33, 20] is designed to minimise the phase error, based on the cost function

$$
\mathcal{J}_{\mathrm{pe}(k)}=\left|e_{\mathrm{pe}}(k)\right|^{2}=|\angle v(k+1)-\angle(v(k) w(k))|^{2}
$$

Its update is given by

$$
w(k+1)=w(k)-\mu \nabla_{w(k)} \mathcal{J}_{\mathrm{pe}(k)}
$$

and from $\nabla_{w(k)} \mathcal{J}_{\mathrm{pe}(k)}=2 e_{\mathrm{pe}}(k) \frac{\partial e_{\mathrm{pe}}(k)}{\partial w^{*}(k)}$ the single-tap weight update of LMP has the form

$$
w(k+1)=w(k)+\frac{\jmath \mu e_{\mathrm{pe}}(k) v^{*}(k)}{(v(k) w(k))^{*}}
$$

Since the estimated instantaneous frequency of a three-phase system is a derivative of the estimated phase, this suits the LMP algorithm, which rotates the filter output towards the desired signal and corrects solely for the phase error. Therefore, for balanced system conditions (and thus a circular system) LMP is expected to achieve enhanced frequency estimation over the standard, error power based, CLMS.

The Least Mean Magnitude Phase (LMMP) based estimation. The LMMP decomposes the cost function of CLMS,
$\mathcal{J}=\frac{1}{2}|e(k)|^{2}$, into a sum of two terms: the magnitude-only term that is minimised when $|v(k+1)|=|\hat{v}(k+1)|$, and the phase-only term that is minimised when $|\angle v(k+1)|=$ $|\angle \hat{v}(k+1)|$, that is [31]

$$
\begin{aligned}
\mathcal{J} & =\underbrace{(|v(k+1)|-|\hat{v}(k+1)|)^{2}}_{\mathcal{J}_{m}(k+1)} \\
& +\underbrace{2|v(k+1)||\hat{v}(k+1)|(1-\cos (\angle v(k+1)-\angle \hat{v}(k+1)))}_{\mathcal{J}_{p}(k+1)}
\end{aligned}
$$

where $\mathcal{J}_{m}(k+1)$ and $\mathcal{J}_{p}(k+1)$ are respectively the magnitude and phase cost functions that inherently exist in the cost function of CLMS. The corresponding gradients are

$$
\begin{aligned}
\nabla_{w^{*}} \mathcal{J}_{m} & =-2(|v(k+1)| \operatorname{sgn}(\hat{v}(k+1))-\hat{v}(k+1)) v^{*}(k) \\
\nabla_{w^{*}} \mathcal{J}_{p} & =-2(v(k+1)-|v(k+1)| \operatorname{sgn}(\hat{v}(k+1))) v^{*}(k)
\end{aligned}
$$

where $\operatorname{sgn}(\hat{v}(k+1))=\hat{v}(k+1) /|\hat{v}(k+1)|$. We can assign different weights to these cost functions. A weighted linear combination of $\mathcal{J}_{m}$ and $\mathcal{J}_{p}$ through different learning rates $\mu_{m}$ and $\mu_{p}$ gives the Least-Mean-Magnitude-Phase (LMMP) update [31]

$$
\begin{aligned}
w(k+1) & =w(k)+ \\
& +\mu_{m}(|v(k+1)| \operatorname{sgn}(\hat{v}(k+1))-\hat{v}(k+1)) v^{*}(k) \\
& +\mu_{p}(v(k+1)-|v(k+1)| \operatorname{sgn}(\hat{v}(k+1))) v^{*}(k)
\end{aligned}
$$

This way we can leverage the magnitude-only and phase-only based estimation and have a whole range of algorithms between the CLMS and LMP.

The ACLMS based frequency estimation. In unbalanced system conditions, the distribution of the voltage in (5) is rotation dependent (noncircular) and the signal is adequately modelled (see the Appendix A) only by using the widely linear model in (13), whose adaptive version is given by

$$
v(k)=A(k) e^{\jmath(\omega k \Delta \mathrm{T}+\phi)}+B(k) e^{-\jmath(\omega k \Delta \mathrm{T}+\phi)}
$$

In other words, when $V_{a}(k), V_{b}(k), V_{c}(k)$ are not identical, $A(k)$ is no longer a constant, $B(k) \neq 0$, and the model in (6) is not adequate. The coefficients of the above widely linear model can be adapted using the Augmented CLMS (ACLMS), given by [37, 36]

$$
\begin{aligned}
\hat{v}(k+1) & =\underbrace{v(k) h(k)}_{\text {standard update }}+\underbrace{v^{*}(k) g(k)}_{\text {conjugate update }} \\
e(k) & =v(k+1)-\hat{v}(k+1) \\
h(k+1) & =h(k)+\mu e(k) v^{*}(k) \\
g(k+1) & =g(k)+\mu e(k) v(k)
\end{aligned}
$$

where $h(k)$ and $g(k)$ are respectively the standard and conjugate filter weights. The stability of ACLMS has been investigated in [41]; the upper bound for the step-size of ACLMS is roughly half that of CLMS. 
From (25) and (26), the estimate $\hat{v}(k+1)$ becomes

$$
\begin{aligned}
\hat{v}(k+1) & =A(k) h(k) e^{\jmath(\omega k \Delta \mathrm{T}+\phi)}+B(k) h(k) e^{-\jmath(\omega k \Delta \mathrm{T}+\phi)} \\
& +A^{*}(k) g(k) e^{-\jmath(\omega k \Delta \mathrm{T}+\phi)}+B^{*}(k) g(k) e^{\jmath(\omega k \Delta \mathrm{T}+\phi)} \\
& =\left(A(k) h(k)+B^{*}(k) g(k)\right) e^{\jmath(\omega k \Delta \mathrm{T}+\phi)} \\
& +\left(A^{*}(k) g(k)+B(k) h(k)\right) e^{-\jmath(\omega k \Delta \mathrm{T}+\phi)}
\end{aligned}
$$

while from $(25), v(k+1)$ can be rewritten as

$$
\begin{aligned}
v(k+1) & =A(k+1) e^{\jmath \omega \Delta \mathrm{T}} e^{\jmath(\omega k \Delta \mathrm{T}+\phi)} \\
& +B(k+1) e^{-\jmath \omega \Delta \mathrm{T}} e^{-\jmath(\omega k \Delta \mathrm{T}+\phi)}
\end{aligned}
$$

Comparing the 'standard', strictly linear parts within (27) and (28), the term $e^{\jmath \omega \Delta T}$ containing the frequency information can be estimated from

$$
e^{\jmath \hat{\omega} \Delta \mathrm{T}}=\frac{A(k) h(k)+B^{*}(k) g(k)}{A(k+1)}
$$

while comparing the conjugate parts within (27) and (28), the evolution of the term $e^{-\jmath \omega \Delta \mathrm{T}}$ can be expressed as

$$
e^{-\jmath \hat{\omega} \Delta \mathrm{T}}=\frac{A^{*}(k) g(k)+B(k) h(k)}{B(k+1)}
$$

thus giving

$$
e^{\jmath \hat{\omega} \Delta \mathrm{T}}=\frac{A(k) g^{*}(k)+B^{*}(k) h^{*}(k)}{B^{*}(k+1)}
$$

The assumption held implicitly in frequency estimation by adaptive filtering algorithms is that, at two consecutive time instants, $A(k+1) \approx A(k)$, and also $B(k+1) \approx B(k)$. This way, (29) and (31) can be respectively simplified into

$$
\begin{aligned}
& e^{\jmath \hat{\omega} \Delta \mathrm{T}}=h(k)+\frac{B^{*}(k)}{A(k)} g(k) \\
& e^{\jmath \hat{\omega} \Delta \mathrm{T}}=h^{*}(k)+\frac{A(k)}{B^{*}(k)} g^{*}(k)
\end{aligned}
$$

Appendix A shows that the coefficient $A(k)$ is real-valued whereas $B(k)$ is complex-valued, and thus $\frac{B^{*}(k)}{A(k)}=\left(\frac{B(k)}{A(k)}\right)^{*}$. Since (32) should be equal to (33), using $a(k)=\left(\frac{B(k)}{A(k)}\right)^{*}$, we can find the expression for $a(k)$ by solving the following quadratic equation with complex-valued coefficients

$$
g(k) a^{2}(k)+\left(h(k)-h^{*}(k)\right) a(k)-g^{*}(k)=0
$$

Since the system frequency is far smaller than the sampling frequency, the correct solution is that for which imaginary part of $e^{\jmath \hat{\omega} \Delta \mathrm{T}}$ is positive, that is

$$
a(k)=\frac{-\jmath \Im(h(k))+\jmath \sqrt{\Im^{2}(h(k))-|g(k)|^{2}}}{g(k)}
$$

and the widely linear system frequency estimate $\hat{f}(k)$ is calculated in the form

$$
\hat{f}(k)=\frac{1}{2 \pi \Delta \mathrm{T}} \sin ^{-1}\left(\Im\left(h(k)+a_{1}(k) g(k)\right)\right)
$$

The above expression is a generic widely linear extension of the standard, strictly linear, frequency estimation method, and can be implemented by any type of widely linear adaptive filter. When the system is balanced, $g(k)=0$, and (36) simplifies into the standard linear solution.

\section{UNBALANCED SYSTEM CONDITIONS AND CLASSIFICA- TION OF VOLTAGE SAGS}

There are seven typical three-phase voltage sags that cause system imbalance; single-phase-to-ground ones are characterised in Table 1, while Table 2 characterises two-phase-toground sags. The corresponding phasor diagrams, together with the associated circularity plots, are given in Fig. 7 and Fig. 8. Observe that apart from the symmetric Type A voltage sag, all the other sags exhibit noncircular amplitude distribution and the frequency drifts are thus expected to be best modelled by widely linear models. The shape, orientation and principal axes are different for the various types of sags, allowing us to identify the fault based on its circularity properties. For instance, Type $\mathrm{C}$ sag is noncircular and aligned horizontally, whereas Type B sag is noncircular and is aligned vertically. Similar observations can be made based on the two-phase-to-ground faults in Fig. 8.

Ambiguities and duality between the sags. Consider a Type B sag, that is, a phase to-ground fault at phasor $v_{a}$ for a starconnected load, shown in Fig. 7. For the same voltage sag in a delta-connected load (where terminal voltages are phase-tophase voltages), the parameters of the corresponding voltage sag are obtained using the star-delta (Yd) transformation

$$
\left[\begin{array}{c}
V_{a}^{\prime} \\
V_{b}^{\prime} \\
V_{c}^{\prime}
\end{array}\right]=\frac{\jmath}{\sqrt{3}}\left[\begin{array}{ccc}
0 & 1 & -1 \\
-1 & 0 & 1 \\
1 & -1 & 0
\end{array}\right]\left[\begin{array}{l}
V_{a} \\
V_{b} \\
V_{c}
\end{array}\right]
$$

where the factor $\sqrt{3}$ keeps the system power invariant, while the $90^{\circ}$ rotation (by the imaginary unit $j$ ) keeps the axis of symmetry of the sag along the real axis. Owing to the rotation and coordinate transformation, a Type B sag in a deltaconnected load can be seen as Type $\mathrm{C}$ sag, usually considered to be caused by a phase-to-phase fault, where the voltage phasors in the two faulted phases move toward each other. After applying (37) once again, the equivalent sag for a phase-tophase connected load becomes a Type D sag.

The equivalent forms for sags due to two phase-to-ground faults can be derived in the same way. For example, for a Type E sag, listed in Table 2, after applying the transformation in (37) we obtain a Type F sag, whereas applying the Yd transformation twice on Type E sag gives Type G sag. Relationships between faults, load connections and sag types are given in Table 3; for more detail see [5]. Most common sags are of Types A, C, and D [27]. Type A sag does not alter the circularity of the complex voltage and for frequency estimation standard linear adaptive estimators (CLMS, LMP, LMMP) are 
Table 1. Four types of voltage sags due to single phase-to-ground fault

\begin{tabular}{|c|c|c|c|}
\hline Type A & Type B & Type C & Type D \\
\hline$V_{a}=V$ & $V_{a}=V$ & $V_{a}=1$ & $V_{a}=V$ \\
$V_{b}=\left(-\frac{1}{2}-\frac{\jmath \sqrt{3}}{2}\right) V$ & $V_{b}=-\frac{1}{2}-\frac{\jmath \sqrt{3}}{2}$ & $V_{b}=-\frac{1}{2}-\frac{\jmath \sqrt{3}}{2} V$ & $V_{b}=-\frac{1}{2} V-\frac{\jmath \sqrt{3}}{2}$ \\
$V_{c}=\left(-\frac{1}{2}+\frac{3 \sqrt{3}}{2}\right) V$ & $V_{c}=-\frac{1}{2}+\frac{j \sqrt{3}}{2}$ & $V_{c}=-\frac{1}{2}+\frac{\jmath \sqrt{3}}{2} V$ & $V_{c}=-\frac{1}{2} V+\frac{\jmath \sqrt{3}}{2}$ \\
\hline
\end{tabular}

Table 2. Three types of voltage sags due to two phase-to-ground faults

\begin{tabular}{|c|c|c|}
\hline Type E & Type F & Type G \\
\hline$V_{a}=1$ & $V_{a}=V$ & $V_{a}=\frac{2}{3}+\frac{V}{3}$ \\
$V_{b}=\left(-\frac{1}{2}-\frac{\jmath \sqrt{3}}{2}\right) V$ & $V_{b}=-\frac{3 \sqrt{3}}{3}-\frac{V}{2}-\frac{\jmath \sqrt{3} V}{6}$ & $V_{b}=-\frac{1}{3}-\frac{V}{6}-\frac{\jmath \sqrt{3} V}{2}$ \\
$V_{c}=\left(-\frac{1}{2}+\frac{\jmath \sqrt{3}}{2}\right) V$ & $V_{c}=\frac{\jmath \sqrt{3}}{3}-\frac{V}{2}+\frac{\jmath \sqrt{3} V}{6}$ & $V_{c}=-\frac{1}{3}-\frac{V}{6}+\frac{\jmath \sqrt{3} V}{2}$ \\
\hline
\end{tabular}
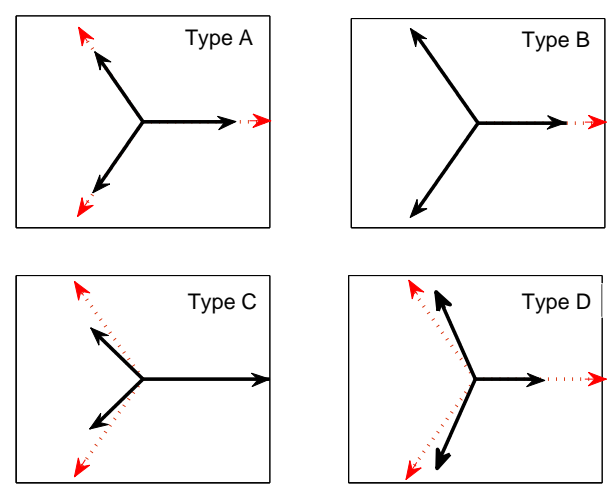

(a) Phasor diagram (dotted line denotes balanced operation)
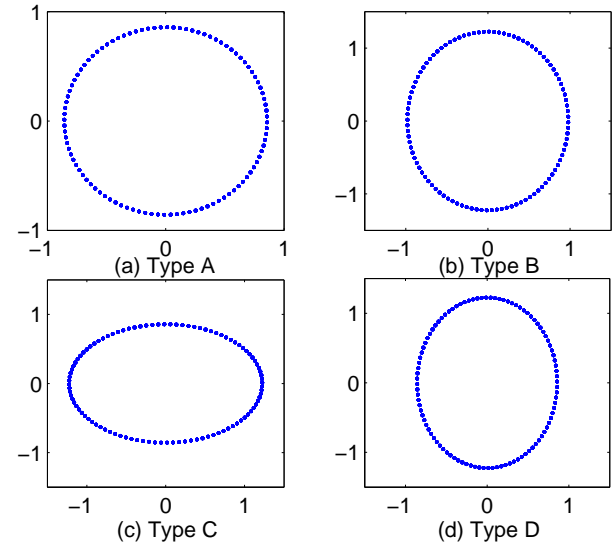

(b) Circularity via a "real-imaginary" plot

Fig. 7. The four unbalanced voltage sags due to single phase-to-ground faults, for the characteristic complex voltage of $V=$ 0.7 .
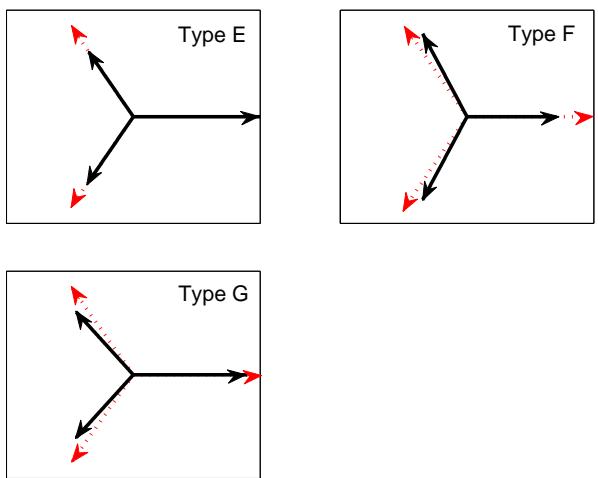

(a) Phasor diagram (dotted line denotes balanced operation)
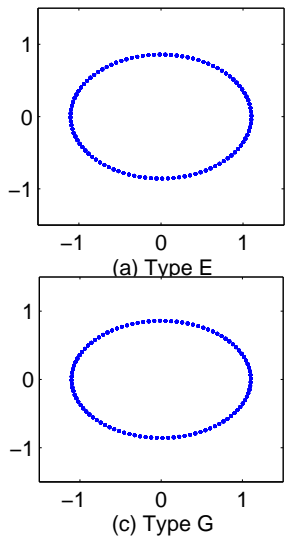

(b) Circularity via a "real-imaginary" plot

Fig. 8. The three unbalanced voltage sags due to two phase-to-ground faults for the characteristic complex voltage of $V=0.7$.

adequate, whereas sags of Type $\mathrm{C}$ and Type $\mathrm{D}$ exhibit noncircular amplitude distributions and the system frequency is thus best estimated using the widely linear ACLMS. From Fig. 7 and 8 , we can observe that when the phase difference between phasors $V_{b}$ and $V_{c}$ is smaller than the nominal $\frac{2}{3} \pi$ (such in the case of Type $\mathrm{C}$ and Type $\mathrm{G}$ sags), the corresponding circularity plots show a vertically aligned ellipse, whereas when the phase difference between phasors $V_{b}$ and $V_{c}$ is larger than 
Table 3. Causes of three-phase voltage sags

\begin{tabular}{|c|c|c|}
\hline Fault type & Load: star & Load: delta \\
\hline Three-phase & Type A & Type A \\
\hline Two-phase-to-ground & Type E & Type F \\
\hline Phase-to-phase & Type C & Type D \\
\hline Single-phase & Type B & Type C \\
\hline
\end{tabular}
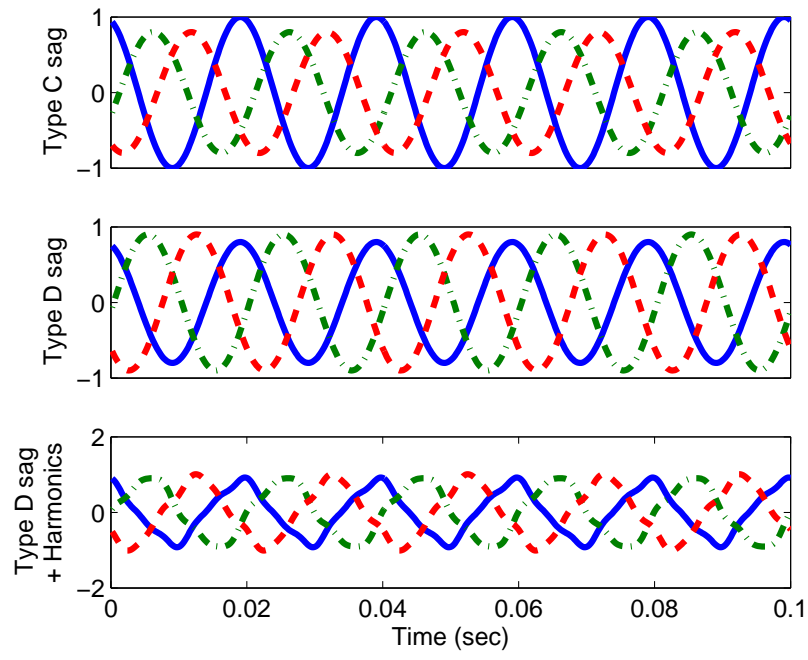

Fig. 9. Time waveforms for different voltage sag types.

$\frac{2}{3} \pi$ (Type D and F sags), a horizontally aligned ellipse is observed. Time waveforms of several typical voltage sags are shown in Fig. 9.

\section{EXAMPLES OF STRICTLY LINEAR AND WIDELY LINEAR FREQUENCY ESTIMATION}

The following case studies are most frequent in practical applications and will be addressed in this section.

- Frequency estimation in the presence of voltage sags, where the system frequency remains at $50 \mathrm{~Hz}$, but due to system imbalance the frequency reading is wrong;

- Frequency rise and decay, which routinely occurs in microgrids and islands, due to a mismatch between production and consumption, resulting in the change in system frequency;

- Presence of harmonics, caused by heavy loads, imbalance of active and reactive power due to the incorporation of intermittent renewable generation, or switching between the main grid and microgrids.

Simulations were performed in the Matlab programming environment, for signals sampled $5 \mathrm{kHz}$, and the step-size $\mu$ of all algorithms was set to be 0.01 in all simulations.

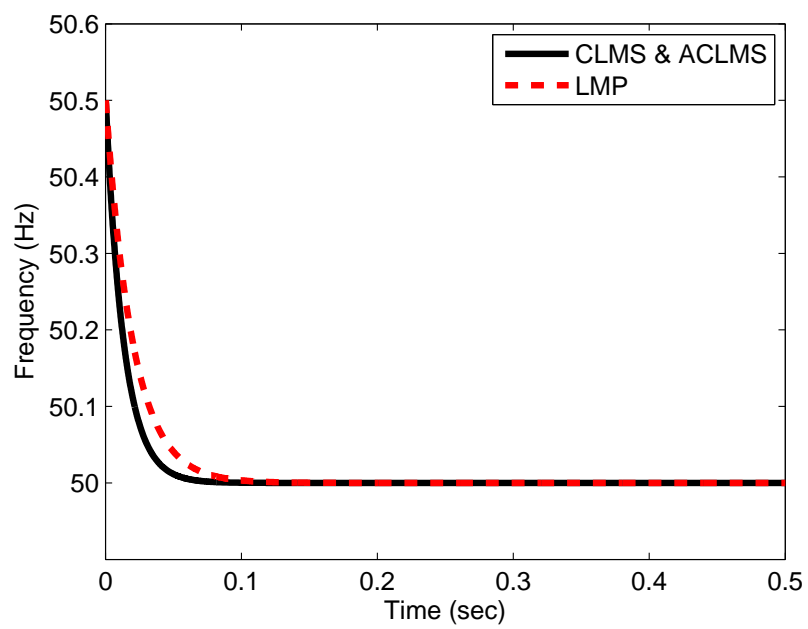

Fig. 10. Illustration of the speed of convergence in frequency estimation for all the strictly linear algorithms for a noise-free balanced power system.

Table 4. percentage errors of all the algorithms in frequency estimation in noisy balanced power system

\begin{tabular}{|c|c|c|c|c|c|}
\hline SNR [dB] & 50 & 40 & 30 & 20 & 10 \\
\hline CLMS & $0.047 \%$ & $0.17 \%$ & $0.53 \%$ & $2.73 \%$ & $14.73 \%$ \\
\hline LMP & $0.032 \%$ & $0.11 \%$ & $0.33 \%$ & $1.05 \%$ & $13.72 \%$ \\
\hline ACLMS & $0.048 \%$ & $0.17 \%$ & $0.53 \%$ & $1.83 \%$ & $13.21 \%$ \\
\hline
\end{tabular}

Balanced system operation. In the first set of simulations, the power system was operating at a system frequency of $f=50 \mathrm{~Hz}$, it was balanced and had distortion-free threephase voltages (normalised to unit magnitude), and all the considered frequency tracking algorithms were initialised with $f_{0}=50.5 \mathrm{~Hz}$. Fig. 10 illustrates the convergence of the adaptive frequency tracking algorithms considered for this circular complex voltage arrangement (see (5)), conforming with the analysis: the CLMS and ACLMS had similar performance and converged faster than LMP, however, the phase-error based LMP exhibited robust performance after convergence. This is further depicted in Table 4, for a system contaminated by noise over a range of Signal to Noise Ratio (SNR) levels.

Frequency estimation in the presence of synthetic voltage sags. Fig. 11 compares performances of the strictly linear CLMS, LMP and LMMP for a system disturbance caused by Type $\mathrm{C}$ voltage sag (with characteristic complex voltage $V=0.7$ ), typical waveforms for a Type $\mathrm{C}$ sag are shown in Fig. 9. Initially, the simulated power system was in its normal operation, at $50 \mathrm{~Hz}$, it had balanced distortion-free three-phase voltages with unit magnitude and all the estimators converged in a similar way. Then at $\mathrm{t}=0.05 \mathrm{~s}$, a Type $\mathrm{C}$ sag occurred, with around a $12 \%$ voltage drop and $9.5^{\circ}$ phase angle offset for phases $v_{b}$ and $v_{c}$, leading to an unbalanced system with a degree of noncircularity $\eta=0.3501$ (Fig. 7). 


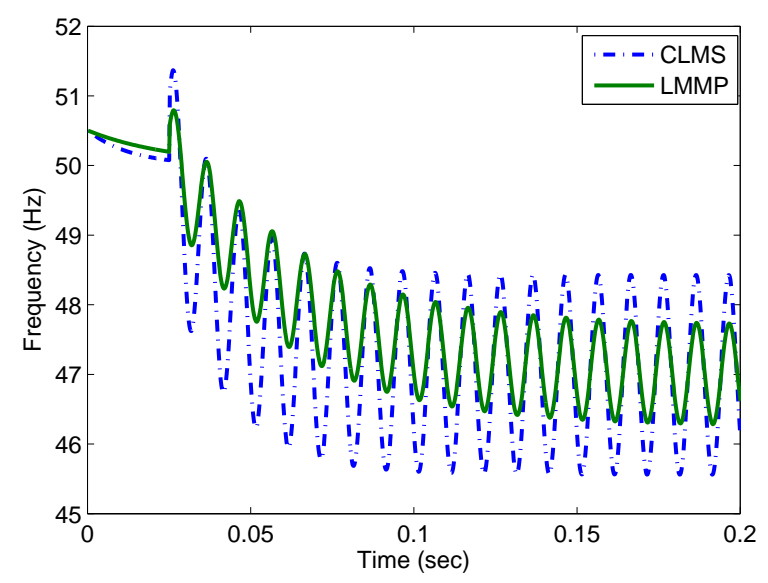

(a) CLMS vs. LMMP - LMMP exhibits lower variance in estimation

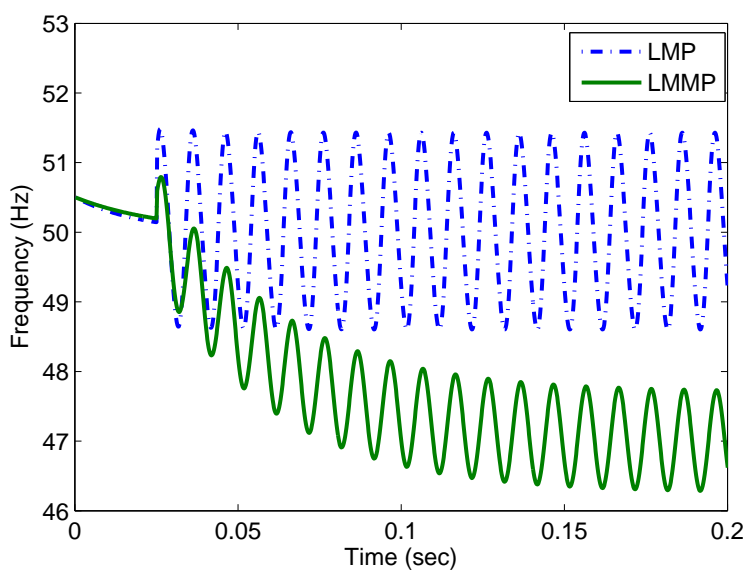

(b) LMP vs. LMMP - LMP has advantage in terms of the bias and LMMP in terms of the error variance

Fig. 11. Comparison of CLMS, LMMP and LMP for frequency estimation in the presence of Type $\mathrm{C}$ voltage sag.

The LMMP algorithm showed smallest variation in frequency estimation whereas the solely phase error based LMP algorithm was the only unbiased algorithm - none of them was optimal for the noncircular unbalanced three-phase system.

Frequency estimation in the presence of harmonics, and for a succession of sags. Fig. 12 illustrates the behaviour of ACLMS, LMP, and CLMS for a system undergoing a sequence of harmful events. The simulated power system was initially in its normal operating mode, at $50 \mathrm{~Hz}$, with balanced distortion-free three-phase voltages of unit magnitudes. Then, at $\mathrm{t}=0.05 \mathrm{~s}$, a Type $\mathrm{C}$ sag occurred, with around a $12 \%$ voltage drop and $9.5^{\circ}$ phase angle offset in phases $v_{b}$ and $v_{c}$, leading to an unbalanced three-phase power system with a degree of noncircularity $\eta=0.3501$ (Fig. 7). There was an inevitable oscillation error at twice of the system frequency for both the CLMS and LMP based estimation due to the undermodelling (see Appendix B). The phase error based LMP al-

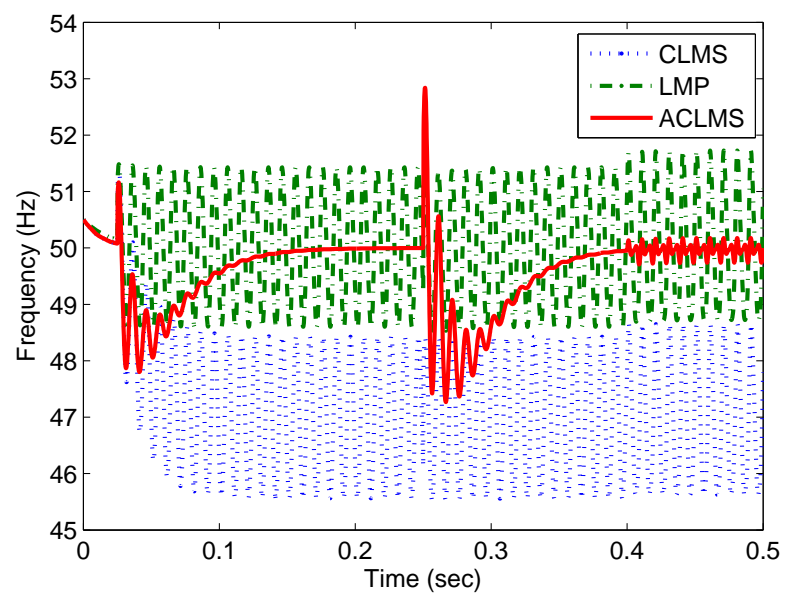

Fig. 12. Frequency estimation using the strictly linear CLMS and widely linear ACLMS (for $\mu=0.01$ ), for frequency estimation in a three phase power system undergoing three consecutive voltage sags of different natures, together with harmonic disturbance.

gorithm eliminated the bias encountered by CLMS, whereas the advantage of the widely linear ACLMS based estimator in accurately estimating the frequency can be seen after convergence (after about $100 \mathrm{~ms}$ ). Then at $\mathrm{t}=0.25 \mathrm{~s}$, a Type $\mathrm{D}$ sag took place exhibiting a $30 \%$ voltage drop for phase $v_{a}$ and $6.6 \%$ voltage drop for both the phases $v_{b}$ and $v_{c}$, together with a $8^{\circ}$ phase angle offset, exhibiting the degree of noncircularity of $\eta=0.3433$. Again, the widely linear ACLMS gave an unbiased performance, whereas the CLMS was not adequate. Finally, after $\mathrm{t}=0.4 \mathrm{~s}$, a $10 \%$ of the third harmonic and $10 \%$ of the fifth harmonic of the fundamental frequency were added into the unbalanced three-phase power system suffering from the same Type D sag to give the circularity index of $\eta=0.3920$. The estimated frequency was subject to an oscillatory steady state error; from $\mathrm{t}=0.4 \mathrm{~s}$, the ACLMS achieved significantly better performance with a smaller oscillation error than the strictly linear CLMS and LMP.

Frequency estimation in the presence of a mismatch between the generation and consumption - frequency rise and decay. Fig. 13 illustrates the superior performance of the widely linear ACLMS over CLMS for a power system experiencing frequency rise and decay. In the simulations, the $50 \mathrm{~Hz}$ fundamental frequency estimation was offset by a Type $\mathrm{D}$ unbalanced three-phase voltage sag, and the frequency rose and decayed at a rate of $5 \mathrm{~Hz} / \mathrm{sec}$. The ACLMS algorithm followed the true system frequency very closely after an initialisation period of around $0.05 \mathrm{sec}$, whereas as expected CLMS produced a biased estimation with large variance.

Theoretical performance bounds in frequency estimation. Bias in frequency estimation is particularly damaging in smart grid applications, as it indicates non-existing shifts in frequency, whereas oscillations (error variance) in the estimate 


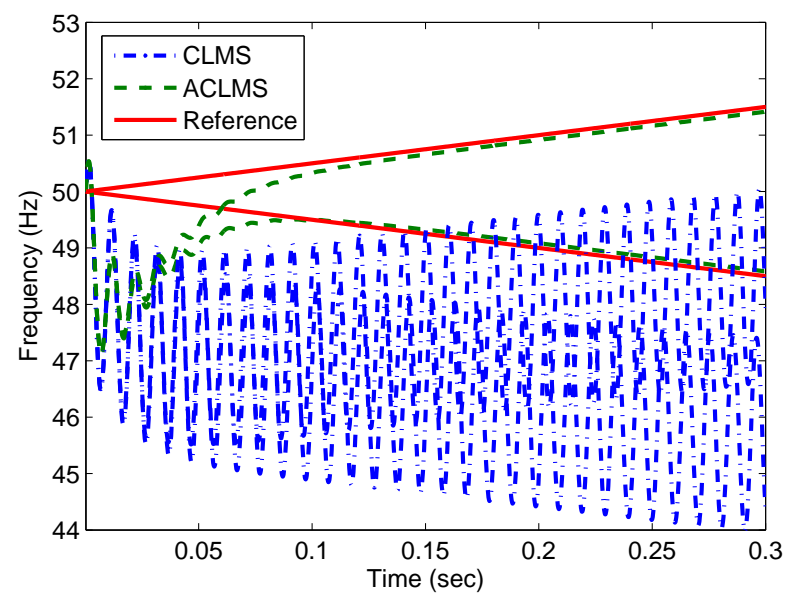

Fig. 13. Frequency rise and decay. Illustration of frequency estimation for a generation - consumption mismatch (microgrids, islanding), when the system frequency is undergoing a decay $(G<L)$ and rise $(G>L)$ at a rate of $5 \mathrm{~Hz} / \mathrm{sec}$. The widely linear ACLMS approached the correct value to within $5 \%$ in 50ms, whereas the strictly linear CLMS could not cope with the unbalanced system conditions. The simulations were conducted for $\mu=0.01$.

indicate that the estimation algorithm is not adequate. A biased estimate will send false alarms through the system, or force the operator to wait for the bias to settle before taking action - both potentially hazardous.

Fig. 14 illustrates the statistical advantage of widely linear frequency estimation over its strictly linear counterpart. Bias and variance analysis for all the algorithms considered were conducted in a noisy environment, by averaging 1000 independent trials. The Cramer-Rao lower bound (CRLB), a theoretical performance bound for the variance of a frequency estimator of a single tone complex exponential contaminated by zero-mean complex-valued doubly white Gaussian noise is derived in Appendix C. Compared with CLMS and LMMP, the phase error based LMP achieved a smaller bias, supporting the analysis. The strictly linear CLMS, LMP, and LMMP were inadequate for unbalanced system conditions (noncircular), they were biased and the bias was not affected by the noise level. The widely linear ACLMS-based estimator was asymptotically unbiased for high SNR.

A similar conclusion can be drawn for the analysis of the estimation variance. Out of the strictly linear algorithms, the LMMP was most robust (also confirmed in Fig. 11), followed by LMP and CLMS. The unavoidable oscillation error experienced by strictly linear estimators, as shown in Fig. 12, gave rise to the high estimation error variance, whereas the widely linear ACLMS estimator was consistent, approaching the CRLB to within $9 \mathrm{~dB}$ in the high SNR region.

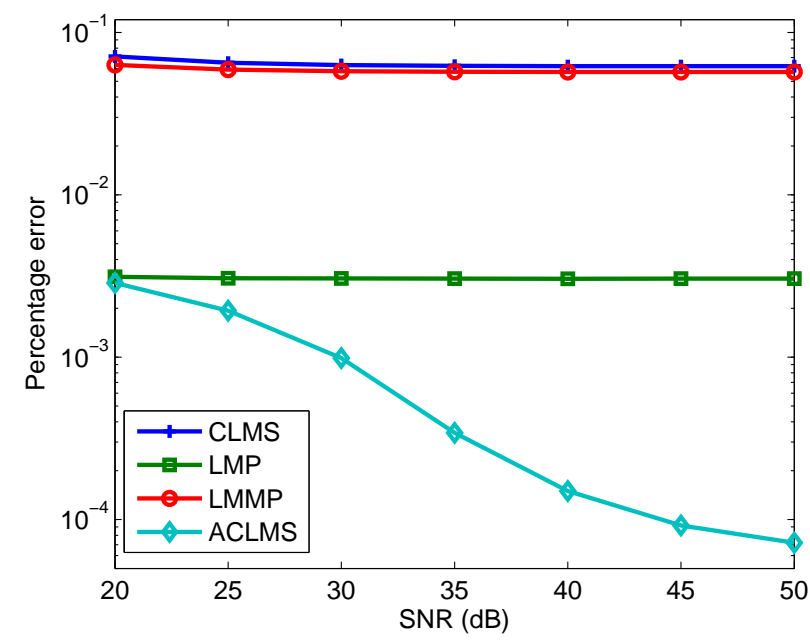

(a) Bias: the widely linear ACLMS is asymptotically unbiased, followed by LMP

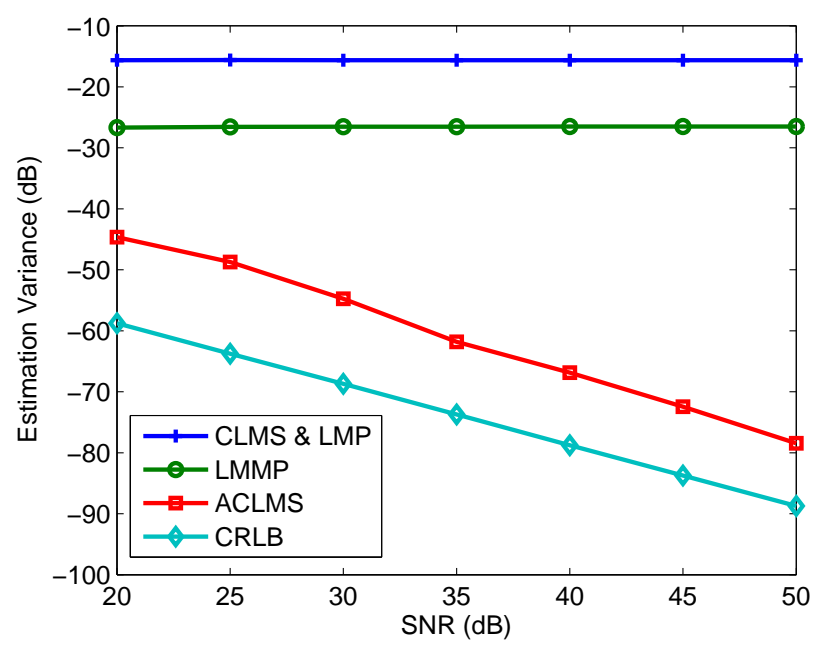

(b) Variance: the widely linear ACLMS is a consistent estimator

Fig. 14. Analysis of bias and variance in frequency estimation for the widely linear ACLMS, and the strictly linear CLMS, LMP and LMMP, relative to theoretical bound for minimum variance unbiased estimation, the Cramer-Rao lower bound (CRLB). The curves are obtained for different SNRs, and averaged over 1000 independent trials.

\section{ESTIMATION OF REAL WORLD VOLTAGE SAGS}

Real-world three phase voltage sags were recorded at a 110/20/10kV transformer station, using the ABB REL 531 numerical line distance protection terminal monitoring 'phaseto-ground' voltages. The device was set to record whenever the phase voltage value dropped below $90 \%$ of its nominal value for longer than $20 \mathrm{~ms}$, and was sampling at $1 \mathrm{kHz}$; the voltage waveforms normalised with respect to their nominal peak values are shown in Fig. 16(a) and (c). 


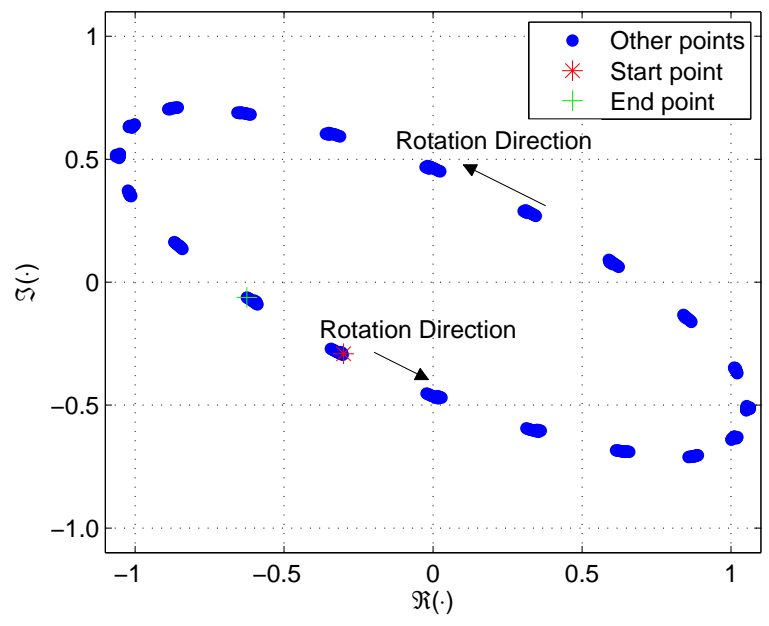

(a) Circularity plot for Case 1

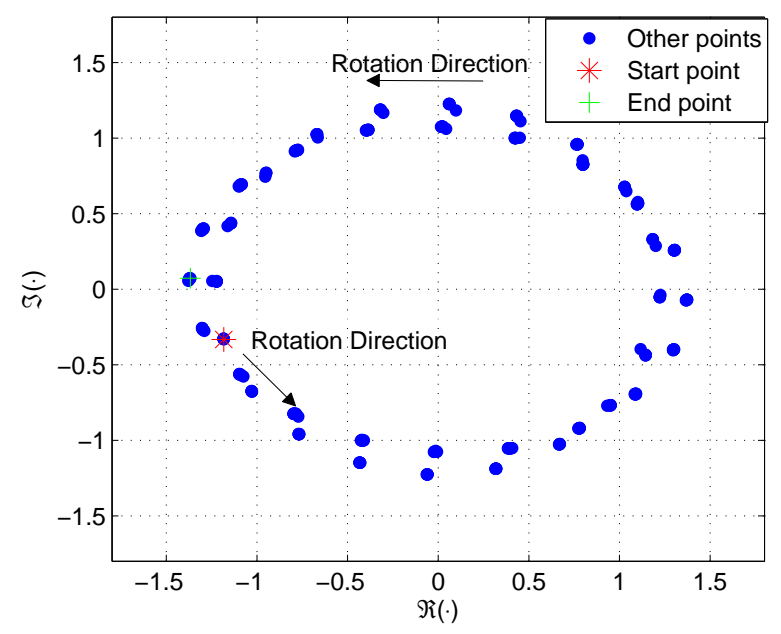

(b) Circularity plot for Case 2

Fig. 15. Noncircularity of real world unbalanced voltage sags via a 'real-imaginary' scatter plot in the complex plane.

In the first case study, a problem in phase $v_{c}$ occurred (shortcut with earth), causing a $94 \%$ voltage drop, while the voltages in phases $v_{a}$ and $v_{b}$ kept their nominal values, to give a degree of noncircularity of $\eta=0.8081$. In the second case study, at around $\mathrm{t}=0.07 \mathrm{sec}$, phase $v_{b}$ experienced a shortcut with earth, resulting in a $65.32 \%$ voltage sag and $79.25 \%$ and $21.92 \%$ voltage swells in phases $v_{a}$ and $v_{c}$ respectively, to give a degree of noncircularity of $\eta=0.2151$. A geometric view of the circularity of the complex voltage $v(k)$ for these two cases is given in Fig. 15 - observe the noncircular natures of these real world problems.

Fig. 16(b) and (d) illustrate the performances of CLMS and ACLMS. Both CLMS and ACLMS were suitable for normal operating conditions; the CLMS was not adequate for the unbalanced situation, whereas the ACLMS could quickly recover and accurately track the true system frequency, which
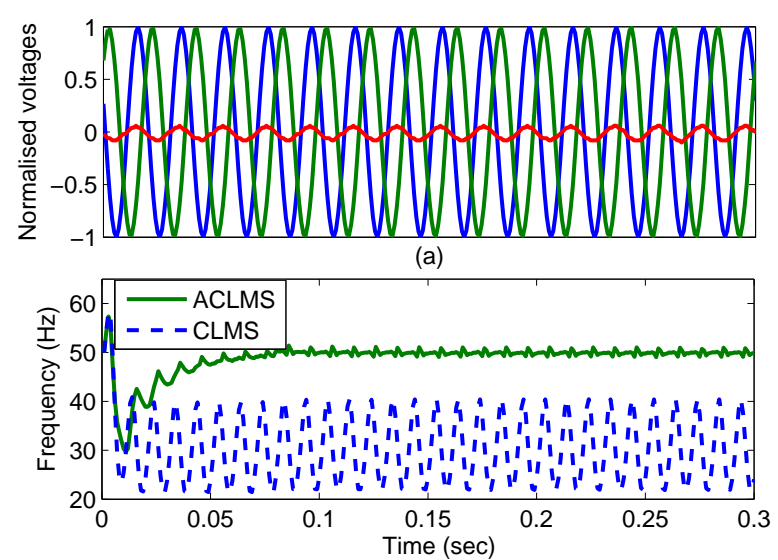

(b)

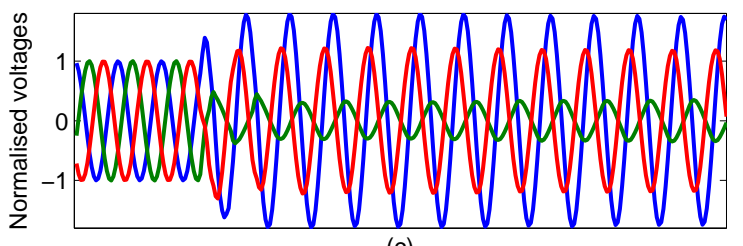

(c)

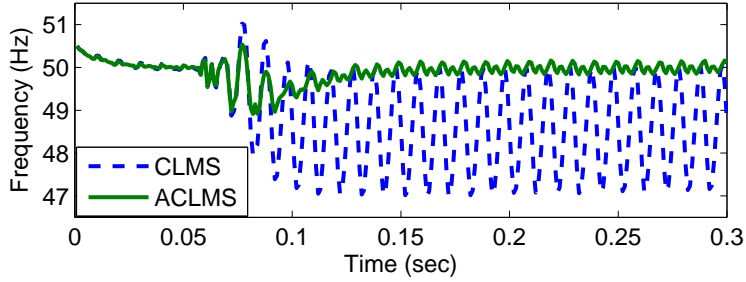

(d)

Fig. 16. Frequency estimation in the presence of real-world voltage sags. (a) Phase C experiences a voltage dip of $94 \%$. (b) Frequency tracking using CLMS and ACLMS. (c) Phase $\mathrm{B}$ experiences a shortcut and phases $\mathrm{A}$ and $\mathrm{C}$ go into a swell. (d) Frequency tracking using CLMS and ACLMS.

remained at $50 \mathrm{~Hz}$.

\section{SUMMARY AND FUTURE OPPORTUNITIES}

We have revisited adaptive frequency estimation in three phase power systems, and have proposed robust frequency estimators suitable for the challenges of smart grid. First, it has been shown that for a balanced system the $\alpha \beta$ transformed three-phase voltage is second order circular (proper), whereas in unbalanced system conditions it is second order noncircular (improper). Circularity plots for voltage sags have indicated the possibility of identifying the type and characteristics of the sag, and the analysis has shown that Type A sag (e.g. motor starting) is proper, while all the other types of voltage sags (Type B - Type G) are improper. Standard strictly linear complex valued estimators are suboptimal for improper data, and a widely linear estimator 
of the instantaneous system frequency has been shown to be second-order optimal for both balanced and unbalanced three-phase systems. The adaptive frequency estimation technique based on the widely linear Augmented Complex Least Mean Square (ACLMS) algorithm has been compared with standard estimation techniques, and has shown excellent tracking ability during dynamic changes in power system, together with reduced sensitivity to higher order harmonics. Unlike the strictly linear frequency estimators based on the CLMS, Least Mean Phase (LMP) and Least Mean Magnitude Phase (LMMP), the ACLMS has been shown to yield unbiased solutions and reduced variance, with a performance improvement over the standard methods that increases with the degree of system imbalance (noncircularity of the phasor). This is suited to smart grid applications, where severe frequency variations are expected due to the on-off switching of various subgrids, and the dual roles of generators and loads (e.g. PEVs).

The use of widely linear modeling in conjunction with Clarke's model increases the number of degrees of freedom, enabling unbiased minimum variance frequency estimation. This has enabled unbiased and minimum variance frequency estimation. Beyond this, it opens opportunities for enhanced grid modeling. For example, by catering for the improperness (second order noncircularity) of the complex $\alpha \beta$ voltage, it is possible to realize:

- Rapid frequency trackers at the distribution level, which are envisaged to be a part of many future appliances (dynamic load, inverters). Smart loads must be able to detect rapid frequency changes and take appropriate action;

- Joint identification and classification of the fault in the system from a voltage dip, based on the different degrees of noncircularity and the shape of the circularity diagram. It is critical that the frequency detector remains accurate during the fault and indicates whether the system experienced one-, two-, or three-phase fault;

- Rate of change frequency trackers, crucial for the operation of microgrids and frequent On/Off switching in the event of islanding;

- Loss-of-mains detection in real time, as a drop in frequency may indicate a loss of a generator and a rise in frequency loss of a load. For instance, the way a wind turbine reacts to a fault depends on the type of voltage sag, and the widely linear methodology enables their identification and tracking at the sub-cycle scale (less than $20 \mathrm{~ms}$ );

- Optimal operation of microgrids - in a cooperative distributed mode, we not only must bring in new generators and interconnect the grid, but also remove low priority loads when power quality deteriorates, also the storage devices that are needed to accommodate the renewable sources compromise the reliability of power quality;

- Low voltage ride through (LVRD) procedures, together with other problems caused by the bidirectional flow of reactive power when renewables are profusely used (e.g. related to the dual role of photovoltaic generators). This may help to further unify and broaden interconnection standards, to address power factor control and LVRD in order to mitigate transient stability issues;

- Forecasting and scheduling, since solar generators and small scale wind turbines are a must-take resource, but are intrinsically intermittent, requiring enhanced realtime control.

\section{ACKNOWLEDGMENTS}

We wish to express our sincere gratitude to Mr. Z. Blazic of Elektroprenos, $\mathrm{BH}$, for providing real world data and expert advice, and to Dr D. Nakafuju of HECO, HI, USA, and Prof T. Green and Prof. G. Strbac from Imperial College London, UK, for fruitful discussions.

\section{APPENDIX A}

From the standard three-phase system in (1) and (4), the components $v_{\alpha}(k)$ and $v_{\beta}(k)$ of the complex voltage $v(k)=$ $v_{\alpha}(k)+\jmath v_{\beta}(k)$, obtained using the $\alpha \beta$ transformation, can be derived as

$$
\begin{aligned}
v_{\alpha}(k) & =\sqrt{\frac{2}{3}}\left(v_{a}(k)-\frac{v_{b}(k)}{2}-\frac{v_{c}(k)}{2}\right) \\
& =\left(\frac{\sqrt{6} V_{a}(k)}{3}+\frac{\sqrt{6}\left(V_{b}(k)+V_{c}(k)\right)}{12}\right) \cos (\omega k \triangle \mathrm{T}+\phi) \\
& -\frac{\sqrt{2}\left(V_{b}(k)-V_{c}(k)\right)}{4} \sin (\omega k \triangle \mathrm{T}+\phi) \\
v_{\beta}(k) & =\sqrt{\frac{2}{3}\left(\frac{\sqrt{3} v_{b}(k)}{2}-\frac{\sqrt{3} v_{c}(k)}{2}\right)} \\
& =-\frac{\sqrt{2}\left(V_{b}(k)-V_{c}(k)\right)}{4} \cos (\omega k \triangle \mathrm{T}+\phi) \\
& +\frac{\sqrt{6}\left(V_{b}(k)+V_{c}(k)\right)}{4} \sin (\omega k \triangle \mathrm{T}+\phi)
\end{aligned}
$$

Given that

$$
\begin{aligned}
& \cos (\omega k \Delta \mathrm{T}+\phi)=\frac{e^{\jmath(\omega k \Delta \mathrm{T}+\phi)}+e^{-\jmath(\omega k \Delta \mathrm{T}+\phi)}}{2} \\
& \sin (\omega k \triangle \mathrm{T}+\phi)=\frac{e^{\jmath(\omega k \Delta \mathrm{T}+\phi)}-e^{-\jmath(\omega k \Delta \mathrm{T}+\phi)}}{2 \jmath}
\end{aligned}
$$

the complex-valued $v(k)$ can be written in the form of a standard part (left hand term) and a conjugate part (right hand 
term) as $v(k)=v_{\alpha}(k)+\jmath v_{\beta}(k)$, that is

$$
v(k)=A(k) e^{\jmath(\omega k \Delta \mathrm{T}+\phi)}+B(k) e^{-\jmath(\omega k \Delta \mathrm{T}+\phi)}
$$

where

$$
\begin{aligned}
& A(k)=\frac{\sqrt{6}\left(V_{a}(k)+V_{b}(k)+V_{c}(k)\right)}{6} \\
& B(k)=\frac{\sqrt{6}\left(2 V_{a}(k)-V_{b}(k)-V_{c}(k)\right)}{12}-\frac{\sqrt{2}\left(V_{b}(k)-V_{c}(k)\right)}{4} \jmath
\end{aligned}
$$

Augmented complex statistics [38, 36] show that $v(k)$ is second order circular with rotation invariant probability density function in the complex plane if $B(k)$ vanishes and $A(k)$ is a constant, which can only be achieved when $V_{a}(k), V_{b}(k), V_{c}(k)$ are identical at each time instant, when (41) simplifies into (6). In unbalanced conditions, $A(k)$ is real-valued, but $B(k) \neq 0$ and can be complex-valued, resulting in a second order noncircular (improper) $v(k)$.

\section{APPENDIX B}

This Appendix gives a theoretical performance analysis on the suboptimality of linear adaptive filters for frequency estimation on unbalanced three-phase voltage systems. In any unbalanced condition, expression (25) stands, and the estimator $\hat{v}(k+1)$, obtained by using the strictly linear modelling based adaptive algorithms, such as CLMS, LMMP, LMP, can be further expressed as

$\hat{v}(k+1)=\left(A(k) e^{\jmath(\omega k \Delta \mathrm{T}+\phi)}+B(k) e^{-\jmath(\omega k \Delta \mathrm{T}+\phi)}\right) w(k)$

In the steady state, $\hat{v}(k+1) \approx v(k+1)$, resulting in

$$
\begin{aligned}
w(k) & =\frac{A(k+1) e^{\jmath(\omega k \Delta \mathrm{T}+\phi)} e^{j \omega \Delta \mathrm{T}}}{A(k) e^{\jmath(\omega k \Delta \mathrm{T}+\phi)}+B(k) e^{-\jmath(\omega k \Delta \mathrm{T}+\phi)}} \\
& +\frac{B(k+1) e^{-\jmath(\omega k \Delta \mathrm{T}+\phi)} e^{-j \omega \Delta \mathrm{T}}}{A(k) e^{\jmath(\omega k \Delta \mathrm{T}+\phi)}+B(k) e^{-\jmath(\omega k \Delta \mathrm{T}+\phi)}}
\end{aligned}
$$

Under the standard assumptions that $A(k+1) \approx A(k)$, and $B(k+1) \approx B(k)$, we have

$$
w(k)=e^{-j \omega \Delta \mathrm{T}}+\frac{e^{j \omega \Delta \mathrm{T}}-e^{-j \omega \Delta \mathrm{T}}}{1+\frac{B(k)}{A(k)} e^{-2 \jmath(\omega k \Delta \mathrm{T}+\phi)}}
$$

where $\frac{B(k)}{A(k)}$ is a unknown parameter, and $w(k)$ is periodic as $w(k)=w\left(k+\frac{1}{2 f \Delta \mathrm{T}}\right)$. In (20), function $\sin ^{-1}$ is a monotonic function, resulting in periodic oscillations in the estimated frequency $\hat{f}(k)$. The cycle frequency due to undermodelling is $2 f$ when using standard linear adaptive filters for frequency estimation of unbalanced power system, whereas for balanced power systems $B(k)=0$, and the standard linear estimate in (20) is adequate.

\section{APPENDIX C}

The widely linear modelling of the complex-valued voltage $v(k)$ obtained from noisy unbalanced three-phase power systems is given by

$$
v(k)=A(k) e^{\jmath(\omega k \Delta \mathrm{T}+\phi)}+B(k) e^{-\jmath(\omega k \Delta \mathrm{T}+\phi)}+n(k)
$$

where $n(k)$ is complex-valued doubly white Gaussian noise with zero mean and variance $\sigma_{n}^{2}$, that is, $n=n_{r}+\jmath n_{i}, \sigma_{n_{r}}^{2}=$ $\sigma_{n_{i}}^{2}=\frac{1}{2} \sigma_{n}^{2}$, and $n_{r} \perp n_{i}$. (44) can be rewritten in terms of its real and imaginary components $v_{r}(k)$ and $v_{i}(k)$ as

$$
\begin{aligned}
v(k) & =\underbrace{(A(k)+B(k)) \cos (2 \pi f k \Delta \mathrm{T}+\phi)+n_{r}(k)}_{v_{r}(k)} \\
& +\jmath \underbrace{\left((A(k)-B(k)) \sin (2 \pi f k \Delta \mathrm{T}+\phi)+n_{i}(k)\right)}_{v_{i}(k)}(45)
\end{aligned}
$$

hence the joint probability density function in terms of doubly white Gaussian noise when the parameter of interest is the system frequency $f$ is given by [42]

$$
\begin{aligned}
p(\boldsymbol{v} ; f) & =\left(\frac{1}{2 \pi \sigma_{n_{r}}^{2}}\right)^{K} \exp \left[-\frac{1}{2 \sigma_{n_{r}}^{2}} \sum_{k=1}^{K}\left(\left(v_{r}(k)-m_{r}(k)\right)^{2}\right.\right. \\
& \left.\left.+\left(v_{i}(k)-m_{i}(k)\right)^{2}\right)\right]
\end{aligned}
$$

where $\boldsymbol{v}=[v(1), v(2), \ldots, v(K)]$ with $K$ the number of observations, $m_{r}(k)=(A(k)+B(k)) \cos (2 \pi f k \Delta \mathrm{T}+\phi)$ and $m_{i}(k)=(A(k)-B(k)) \sin (2 \pi f k \Delta \mathrm{T}+\phi)$. Therefore,

$$
\begin{aligned}
\frac{\partial \ln (p(\boldsymbol{v} ; f))}{\partial f} & =\frac{1}{\sigma_{n_{r}}^{2}} \sum_{k=1}^{K}\left(\left(v_{r}(k)-m_{r}(k)\right) \frac{\partial m_{r}(k)}{\partial f}\right. \\
& \left.+\left(v_{i}(k)-m_{i}(k)\right) \frac{\partial m_{i}(k)}{\partial f}\right)
\end{aligned}
$$

and

$E\left[\frac{\partial^{2} \ln (p(\boldsymbol{v} ; f))}{\partial f^{2}}\right]=-\frac{1}{\sigma_{n_{r}}^{2}} \sum_{k=1}^{K}\left(\left(\frac{\partial m_{r}(k)}{\partial f}\right)^{2}+\left(\frac{\partial m_{i}(k)}{\partial f}\right)^{2}\right)$

Since

$$
\begin{aligned}
& \frac{\partial m_{r}(k)}{\partial f}=-2 \pi k \Delta \mathrm{T}(A(k)+B(k)) \sin (2 \pi f k \Delta \mathrm{T}+\phi) \\
& \frac{\partial m_{i}(k)}{\partial f}=2 \pi k \Delta \mathrm{T}(A(k)-B(k)) \cos (2 \pi f k \Delta \mathrm{T}+\phi)(48)
\end{aligned}
$$

and $\sigma_{n_{r}}^{2}=\frac{1}{2} \sigma_{n}^{2}$. According to the CRLB calculation [43], that is,

$$
\operatorname{var}(\hat{f}) \geq \frac{1}{-E\left[\frac{\partial^{2} \ln (p(\mathbf{v} ; \theta))}{\partial f^{2}}\right]}
$$

The CRLB of frequency estimator on unbalanced three-phase power systems thus can be obtained in (50). 


$$
\operatorname{var}(\hat{f}) \geq \frac{\sigma_{n}^{2}}{2 \sum_{k=1}^{K}(2 \pi k \Delta \mathrm{T})^{2}\left((A(k)+B(k))^{2} \sin ^{2}(2 \pi f k \Delta \mathrm{T}+\phi)+(A(k)-B(k))^{2} \cos ^{2}(2 \pi f k \Delta \mathrm{T}+\phi)\right)}
$$

\section{REFERENCES}

[1] "Section 1304 Smart Grid RD\&D Highlights," USA Energy Independence and Security Act, 2007.

[2] "The cost of power disturbances to industrial and digital economy companies," EPRI, Palo Alto, CA, EPRI Executive Summary 1006274.

[3] K. Lee, G. Venkataramanan, and T. M. Jahns, "Source current harmonic analysis of adjustable speed drives under input voltage unbalance and sag conditions," IEEE Transactions on Power Delivery, vol. 21, no. 2, pp. 567-576, 2006.

[4] J. L. Duran-Gomez, P. N. Enjeti, and B. O. Woo, "Effect of voltage sags on adjustable speed drives - a critical evaluation and approach to improve its performance," Proceedings of 14th Annual Applied Power Electronics Conference and Exposition, vol. 2, pp. 774-780, 1999.

[5] M. H. J. Bollen, Understanding Power Quality Problems Voltages and Interruptions, New York: IEEE Press, 1999.

[6] S. Chakrabarti, E. Kyriakides, T. Bi, D. Cai, and V. Terzija, "Measurements get together," IEEE Power \& Energy Magazine, vol. 7, no. 1, pp. 41-49, 2009.

[7] M. S. Sachdev and M. M. Giray, "A least square technique for determining power system frequency," IEEE Transactions on Power Apparatus and Systems, vol. PAS-104, no. 5, pp. 10251038, 1983.

[8] O. Vainio and S. Ovaska, "Digital filtering for robust $50 / 60 \mathrm{~Hz}$ zero-crossing detecters," IEEE Transactions on Instrumentation and Measurement, vol. 45, no. 2, pp. 426-430, 2001.

[9] T. Lin, M. Tsuji, and E. Yamada, "A wavelet approach to real time estimation of power system frequency," Proceedings of 40th Annual Conference of the Society of Instrument and Control Engineers (SICE), pp. 58-65, 2001.

[10] J. Yang and C. Liu, "A precise calculation of power system frequency," IEEE Transactions on Power Delivery, vol. 16, no. 3, pp. 361-366, 2001.

[11] L. G. B. Barbosa Rolim, Jr. D. R. Rodrigues da Costa, and M. Aredes, "Analysis and software implementation of a robust synchronizing PLL circuit based on the pq theory," IEEE Transsactions on Industrial Electronics, vol. 53, no. 6, pp. 1919-1926, 2006.

[12] V. Eckhardt, P. Hippe, and G. Hosemann, "Dynamic measuring of frequency and frequency oscillations in multiphase power systems," IEEE Transactions on Power Delivery, vol. 4, no. 1, pp. 95-102, 1989.

[13] E. Clarke, Circuit Analysis of A.C. Power Systems, New York: Wiley, 1943.

[14] M. H. J. Bollen, I. Y. H. Gu, S. Santoso, M. F. McGranaghan, P. A. Crossley, M. V. Ribeiro, and P. F. Ribeiro, "Bridging the gap between signal and power," IEEE Signal Processing Magazine, vol. 26, no. 4, pp. 11-31, 2009.
[15] D. E. Bakken, A. Bose, C. H. Hauser, D. E. Whitehead, and G. C. Zweigle, "Smart generation and transmission with coherent, real-time data," Proceedings of the IEEE, vol. 99, no. 6, pp. 928-951, 2011.

[16] P. Rodriguez, J. Pou, J. Bergas, J. I. Candela, R. P. Burgos, and D. Boroyevich, "Decoupled double synchronous reference frame PLL for power converter control," IEEE Transactions on Power Electronics, vol. 22, no. 2, pp. 584-592, 2007.

[17] A. K. Pradhan, A. Routray, and A. Basak, "Power system frequency estimation using least mean square technique," IEEE Transactions on Power Delivery, vol. 20, no. 3, pp. 761-766, 2005.

[18] P. K. Dash, A. K. Pradhan, and G. Panda, "Frequency estimation of distorted power system signals using extended complex Kalman filter,' IEEE Transactions on Power Delivery, vol. 14, no. 3, pp. 761-766, 1999.

[19] M. Akke, "Frequency estimation by demodulation of two complex signals," IEEE Transactions on Power Delivery, vol. 12, no. 1, pp. 157-163, 1997.

[20] T. K. Rawat and H. Parthasarathy, "A continuous-time least mean-phase adaptive filter for power frequency estimation," Electrical Power and Energy Systems, vol. 31, pp. 111-115, 2009.

[21] D. Beeman, Industrial Power System Handbook, McGrawHill, 1955.

[22] H. S. Song and K. Nam, "Instantaneous phase-angle estimation algorithm under unbalanced voltage-sag conditions," IEE Proceedings of Generation, Transmission and Distribution, vol. 147, no. 6, pp. 409-415, 2000.

[23] M. D. Kušljević, J. J. Tomić, and L. D. Jovanović, "Frequency estimation of three-phase power system using weighted-leastsquare algorithm and adaptive FIR filtering," IEEE Transactions on Instrumentation and Measurement, vol. 57, no. 10, pp. 322-329, 2010.

[24] M. Mojiri, D. Yazdani, and A. Bakhshai, "Robust adaptive frequency estimation of three-phase power system," IEEE Transactions on Instrumentation and Measurement, vol. 59, no. 7, pp. 1793-1802, 2010.

[25] M. De Nigris, I. Gianinoniand S. Grillo, S. Massucco, and F. Silvestro, "Impact evaluation of plug-in electric vehicles (PEV) on electric distribution networks," Proceedings of the 14th International Conference on Harmonics and Quality in Power (ICHQP), pp. 1-6, 2010.

[26] A. Ipakchi and F. Albuyeh, "Grid of the future," IEEE Power \& Energy Magazine, vol. 7, no. 2, pp. 52-62, 2009.

[27] M. H. J. Bollen, "Voltage sags in three-phase systems," IEEE Power Engineering Review, vol. 21, no. 9, pp. 8-15, 2001.

[28] J. Charles Smith, R. Thresher, R. Zavadil, E. DeMeo, R. Piwko, B. Ernst, and T. Ackermann, "A mighty wind," IEEE Power \& Energy Magazine, vol. 7, no. 2, pp. 41-51, 2009. 
[29] J. Bebic, R. Wawlling, K. O'Brien, and B. Kroposki, "The sun also rises," IEEE Power and Energy Magazine, vol. 7, no. 3, pp. 34-44, 2009.

[30] L. Zhang and M. H. J. Bollen, "Characterisation of voltage dips in power systems," IEEE Transactions on Power Delivery, vol. 15 , no. 2, pp. 827-832, 2000.

[31] S. C. Douglas and D. P. Mandic, "The least-mean-magnitudephase algorithm with applications to communications systems," Proceedings of ICASSP, pp. 4152-4155, 2011.

[32] B. Widrow, J. McCool, and M. Ball, "The complex LMS algorithm," Proceedings of IEEE, vol. 63, pp. 719-720, 1974.

[33] A. Tarighat and A. H. Sayed, "Least mean-phase adaptive filters with application to communications systems," IEEE Signal Processing Letters, vol. 11, no. 2, pp. 220-223, 2004.

[34] B. Picinbono and P. Chevalier, "Widely linear estimation with complex data," IEEE Transactions on Signal Processing, vol. 43, no. 8, pp. 2030-2033, 1995.

[35] P. J. Schreier and L. L. Scharf, "Second-order analysis of improper complex random vectors and process," IEEE Transactions on Signal Processing, vol. 51, no. 3, pp. 714-725, 2003.

[36] D. P. Mandic and S. L. Goh, Complex Valued Nonlinear Adaptive Filters: Noncircularity, Widely Linear and Neural Models, John Wiley \& Sons, 2009.

[37] S. Javidi, S. L. Goh M. Pedzisz, and D. P. Mandic, "The augmented complex least mean square algorithm with application to adaptive prediction problems," Proceedings of 1st IARP Workshop on Cognitive Information Processing, pp. 5457, 2008.

[38] B. Picinbono, "On circularity," IEEE Transactions on Signal Processing, vol. 42, no. 12, pp. 3473-3482, 1994.

[39] E. Ollila, "On the circularity of a complex random variable," IEEE Signal Processing Letters, vol. 15, pp. 841-844, 2008.

[40] B. Jelfs, D. P. Mandic, and S. C. Douglas, "An adaptive approach for the identification of improper complex signals," Signal Processing, p. tba, in press, 2011.

[41] S. C. Douglas and D. P. Mandic, "Performance analysis of the conventional complex LMS and augmented complex LMS algorithms," Proceedings of IEEE International Conference on Acoustics, Speech, and Signal Processing, pp. 3794-3797, 2010.

[42] D. C. Rife and R. R. Boorstyn, "Single-tone parameter estimation from discrete-time observations," IEEE Transactions on Information Theory, vol. 20, no. 5, pp. 591-598, 1974.

[43] S. M. Kay, Fundamentals of Statistical Signal Processing: Estimation Theory, New Jersey: Prentice Hall, 1993. 